Proyecciones Journal of Mathematics

Vol. 29, No 3, pp. 247-293, December 2010.

Universidad Católica del Norte

Antofagasta - Chile

\title{
BIFURCATION OF THE ESSENTIAL DYNAMICS OF LORENZ MAPS ON THE REAL LINE AND THE BIFURCATION SCENARIO FOR LORENZ LIKE FLOWS : THE CONTRACTING CASE *
}

\author{
RAFAEL LABARCA \\ UNIVERSIDAD DE SANTIAGO DE CHILE, CHILE \\ and \\ CARLOS MOREIRA \\ I. M. P. A., BRASIL \\ Received: October 2010. Accepted : November 2010
}

\begin{abstract}
In this article we provide, by using kneading sequences, the combinatorial bifurcation diagram associated to a typical two parameter family of contracting Lorenz maps on the real line. We apply these results to two parameter families of geometric Lorenz-like flows.
\end{abstract}

Key words : Lorenz maps; combinatorial bifurcation diagramme; lexicographical world, geometric Lorenz-like flows.

Mathematics Subject Classification 2010 : Primary: 37010, 37C10, 37E05. Secondary : 37C20.

\footnotetext{
${ }^{*}$ Partially supported by Fondecyt grants \# 1970720 and \# 1000098, DICYT-USACHChile, PRONEX on Dynamical Systems, Brazil and MATHAMSUD 09MATH02
} 


\section{Introduction}

In a remarkable contribution, the meteorologist E. N. Lorenz [27] exhibited numerical evidence for the existence of a strange attractor in a quadratic system of ordinary differential equations in three variables. Some time later Afrajmovich, Bykov and Shilnikov( [1], [2]) and Guckenheimer, Williams( [15], [40], [16]) proposed the so called geometrical models for the behavior observed by Lorenz. An important feature of these models is the existence of a(partial) cross-section to the flow, as well as a smooth invariant foliation by curves. Using this, one can reduce the dynamics of the flow to that of an interval transformation with a discontinuity. These transformation, generically, divide into two disjoint classes: the expanding ones(those whose derivative, from both sides, at the discontinuity is infinity) and the contracting ones(those whose derivative, from both sides, at the discontinuity is zero). As observed in [16] and [2], there exist uncountably many conjugacy classes of such transformations. In fact the moduli space is essentially 2-dimensional and can be parameterized by the admissible kneading sequences(forward itineraries of the discontinuity).

In view of these results, it is natural to look for a bifurcation theory of these transformations and flows using symbolic dynamics([5, 21, 24]. In this direction, de Melo and Martens showed([9]) the existence of parameterized families of contracting Lorenz flows that are topologically universal in the sense that given any geometric Lorenz flow, its dynamics is "essentially" the same as the dynamics of some element of the family. In the present work we describe in "almost" complete way the bifurcation diagram for an explicit natural choice of such an essentially universal family, namely the quadratic family

$$
F_{\mu, \nu}(x)=\left\{\begin{array}{c}
-\mu+x^{2}, x>0 \\
\nu-x^{2}, x<0
\end{array}\right\}
$$

That is, for this family we give a complete description of the subsets of the plane associated with kneading sequences $(a, b) \in L W$ (see section 2.4 for the definition of the set LW). In other word, in the present paper we give the combinatorial bifurcation pattern associated to our quadratic family(see also $[21,22,23]$.In the way of doing this we first gave the structure of the lexicographical world which seems to be the natural space of parameters for discontinuous maps like the one's that we consider here( see [25], [26]).

Our geometrical construction has several important consequences.One of them is that axiom A maps are dense in this family (this is also proved in 
[9]; see [14] for similar result for the logistic family and [19] for contracting singular cycles). It also follows that the set of parameters whose corresponding Lorenz map has entropy zero is arc connected( see section 4.6) - Also, the combinatorial bifurcation diagram allow us to reobtain, in a constructive way, the result of the Melo and Martens on the universality of the quadratic family. Moreover, we completely describe the exceptional combinatorics not realized in the model(a countable set)provided by de Melo and Martens([9]). Also, as lemma 4 in section 3.1 shows,secondary bifurcations do not appear for injective Lorenz maps( as defined in section 2.1)

After this paper had been completed we became aware of the work of St. Pierre( [37])about the bifurcation diagram associated to periodic kneading sequences, which obtains, in an independent way, some of the results presented here. Also, Hubbard and Sparrow defined, in [18], a set of pair of sequences which model all the topological expanding dynamics exhibited by Lorenz maps.

On the other hand, starting with Arnold ([3]) there has been considerable interest in the bifurcation theory of the canonical family( see for instance [6] and the references there in). The most common approach, based on the notion of rotation number, is very useful when applied to homeomorphism of the circle, but it seems delicate to apply it to non injective maps. In fact, a bifurcation diagram for the canonical family beyond the injectivity domain is still unknown. The best approximation seems to be the work by Boyland $([6])$.

In $[21,22,23]$, we extended the Hubbard-Sparrow model for expanding maps to an universal model for the "essential dynamics" of Lorenz maps and we called it the Lexicographical World (in the sequel denoted by $L W$ ). This model includes the dynamics of all the interpolated maps used by Boyland ([6], page 359 ) in his construction.

It is clear that the structure of the lexicographical world has to reflect in the bifurcation theory associated to any parameterized family of allowed maps. This is an extremely interesting problem focused for several authors in this and other contexts, see for instance [28],[8],[7],[11], [3], [6] .

This paper is organized as follows : In section 2 we state our results. In section 3 we describe the lexicographical world, in Section 4 we prove our results about the quadratic family $F_{\mu, \nu}$, in section 5 we extend our results to more general contracting families and, finally, in section 6 we will relate them to three dimensional geometric vector fields. 


\section{Statement of the results}

\subsection{The Set $D M_{0}$.}

In the sequel $D M_{0}$ will denote the set of maps $f:(R \backslash\{0\}) \rightarrow R$ such that:

(1) The restriction maps $\left.f\right|_{(-\infty, 0)}:(-\infty, 0) \mapsto R$ and $\left.f\right|_{(0, \infty)}:(0, \infty) \rightarrow$ $R$ are continuous and non-decreasing maps.

$(2)$

$$
\left.\left.f\left(0^{+}\right)=\lim _{x \downarrow 0} f(x) \in\right]-\infty, 0\right]
$$

and

$$
f\left(0^{-)}=\lim _{x \uparrow 0} f(x) \in[0, \infty[.\right.
$$

An element in $D M_{0}$ will be called injective if its restriction to the interval $] f\left(0^{+}\right), f\left(0^{-}\right)$is an injective map.

We will say that $f \in D M_{0}$ is increasing if the restriction maps $\left.f\right|_{(-\infty, 0)}$ : $(-\infty, 0) \mapsto R$ and $\left.f\right|_{(0, \infty)}:(0, \infty) \rightarrow R$ are increasing.

We call the elements in $D M_{0}$ Lorenz maps.

\subsection{The Lexicographical Order}

Let $\Sigma_{2}$ denote the set of sequences $\theta: N \rightarrow\{0,1\}$ endowed with the topology given by the metric

$$
d(\alpha, \beta)=\sum_{i=0}^{\infty} \frac{\bar{d}\left(\alpha_{i}, \beta_{i}\right)}{2^{i}},
$$

where

$$
\bar{d}\left(\alpha_{i}, \beta_{i}\right)=\left\{\begin{array}{c}
0, \alpha_{i}=\beta_{i} \\
1, \alpha_{i} \neq \beta_{i}
\end{array}\right.
$$

Let $\sigma: \Sigma_{2} \rightarrow \Sigma_{2}$ be the shift map $\sigma\left(\theta_{0}, \theta_{1}, \theta_{2}, \ldots\right)=\left(\theta_{1}, \theta_{2}, \ldots\right)$. Let $\Sigma_{0}$ and $\Sigma_{1}$ denote the sets $\left\{\theta \in \Sigma_{2} ; \theta_{0}=0\right\}$ and $\left\{\theta \in \Sigma_{2} ; \theta_{0}=\right.$ 1 ) respectively. It is clear that $\Sigma_{2}=\Sigma_{0} \cup \Sigma_{1}$.

In $\Sigma_{2}$ we consider the lexicographical order: $\theta<\alpha$ for any $\theta \in \Sigma_{0}$ and $\alpha \in$ $\Sigma_{1}$ or $\theta<\alpha$ if there is $\overline{n \in N \text { such that } \theta_{i}}=\alpha_{i}$ for $i=0,1,2, \ldots, n-$ 1 and $\theta_{n}=0$ and $\alpha_{n}=1$.

For $a \leq b$ in $\Sigma_{2}$ let $[a, b]$ denote the interval $\left\{\theta \in \Sigma_{2} \mid a \leq \theta \leq\right.$ $b\}$. $\quad \Sigma_{a, b}$ will denote the set $\bigcap_{n=0}^{\infty} \sigma^{-n}([a, b])$. 


\subsection{The Set $\Sigma_{a_{f}, b_{f}}$.}

For $f \in D M_{0}$ let $\Gamma_{f}=\left(R \backslash \bigcup_{j=0}^{\infty} f^{-j}(0)\right)$ denote the set of "continuity" of the map $f$.

For $x \in \Gamma_{f}$ we define $I_{f}(x) \in \Sigma_{2}$ by

$$
I_{f}(x)(i)=0 \quad \text { if } f^{i}(x)<0 \text { and } I_{f}(x)(i)=1 \quad \text { if } f^{i}(x)>0 .
$$

For $x=0$ we define:

$$
I_{f}\left(0^{+}\right)=\lim _{x \downarrow 0, x \in \Gamma_{f}} I_{f}(x)
$$

and

$$
I_{f}\left(0^{-}\right)=\lim _{x \uparrow 0, x \in \Gamma_{f}} I_{f}(x) .
$$

In the same way: to any $x \in \bigcup_{j=0}^{\infty} f^{-j}(0)$ such that $f^{i}(x) \neq 0,0 \leq i<n$; $f^{n}(x)=0$ we associate the sequences :

$$
I_{f}\left(x^{+}\right)=\left(I_{f}(x)(0), \ldots, I_{f}(x)(n-1), I_{f}\left(0^{+}\right)\right)
$$

and

$$
I_{f}\left(x^{-}\right)=\left(I_{f}(x)(0), \ldots, I_{f}(x)(n-1), I_{f}\left(0^{-}\right)\right) .
$$

For $x \in \Gamma_{f}$ we define $I_{f}\left(x^{+}\right)=I_{f}\left(x^{-}\right)=I_{f}(x)$.

Let $I_{f}=\left\{I_{f}\left(x^{+}\right) ; \quad x \in\left[f\left(0^{+}\right), f\left(0^{-}\right)[\} \cup\left\{I_{f}\left(x^{-}\right) ; \quad x \in\right] f\left(0^{+}\right), f\left(0^{-}\right)\right]\right\}$.

Clearly $\sigma\left(I_{f}\right) \subset I_{f}$. Let us denote $a_{f}=I_{f}\left(\left(f\left(0^{+}\right)\right)^{+}\right)$and $b_{f}=I_{f}\left(\left(f\left(0^{-}\right)\right)^{-}\right)$

Lemma 1. ([20], [18]) $I_{f}=\bigcap_{n=0}^{\infty} \sigma^{-n}\left(\left[a_{f}, b_{f}\right]\right)=\Sigma_{a_{f}, b_{f}}$.

We observe that associated to any $f \in D M_{0}$ we can define a continuous map

$$
h:\left[f\left(0^{+}\right), f\left(0^{-}\right)\right] \cap \Gamma_{f} \rightarrow \Sigma_{a_{f}, b_{f}} \subset \Sigma_{2},
$$

such that $h \circ f=\sigma \circ h$. The map $h$ is given by $h(x)=I_{f}(x)$ and collapses intervals into points. This map cannot be extended, continuously, to the set $\cup_{i=0}^{\infty} f^{-i}(0)$. There are two kinds of intervals that the map $h$ can collapses: The wandering intervals and the intervals that are contained in the stable manifold of periodic sinks. An interval $I \subset\left[f\left(0^{+}\right), f\left(0^{-}\right)\right]$is called a wandering interval, for the map $f$, if for any $x \in I$ we have that $x$ is a wandering point. We will call a point $x$ a nonwandering point if for any neighborhood $U_{x}$ of $x$ and any positive integer $N$ we can find $n \geq N$ such that $f^{n}\left(U_{x}\right) \cap U_{x} \neq \emptyset$. The set of nonwandering points of the map $f$ is 
denoted by $\Omega_{f}$. A point $x \notin \Omega_{f}$ is called a wandering point. We call a periodic point $x \in I$, a sink if there is a neighborhood $V_{x} \subset I$ of $x$ such that $\left|f^{n}(x)-f^{n}(y)\right| \rightarrow 0$ as $n \rightarrow \infty$ for any $y \in V_{x}$.

Given any interval , $I$, the orbit of this interval is the sequence of iterations $\left(f^{n}(I), n \in N\right)$. Concerning the existence of wandering intervals we have the following:

Lemma 2. ([23]) Let $\left\{\varphi_{\lambda}, \lambda \in R\right\} \subset D M_{0}$ be a one parameter family of $C^{2}$ increasing maps such that for each $\lambda$ there are sequences $\lambda_{n} \rightarrow \lambda$ and $\mu_{n} \rightarrow \lambda$ with $\varphi_{\lambda_{n}}(x)>\varphi_{\lambda}(x)$ and $\varphi_{\mu_{n}}(x)<\varphi_{\lambda}(x), \forall x$ then there is a residual set of parameters $\lambda$ for which $\varphi_{\lambda}$ has no wandering intervals.

We observe that for our two parameter family of contracting maps, $F_{(\mu, \nu)}$, we have the following: for any fixed $\mu=\mu_{0}>0\left(\right.$ or $\left.\nu=\nu_{0}>0\right)$ the one parameter family $\varphi_{\nu}=F_{\left(\mu_{0}, \nu\right)}\left(\right.$ or $\left.\quad \varphi_{\mu}=F_{\left(\mu, \nu_{0}\right)}\right)$ satisfies the property of Lemma 2.

Definition 1. Given $f, g \in D M_{0}$. We will say that $f$ has essentially the same dynamics as $g$ if $I_{f}=I_{g}$.

We note that in this situation, up to the existence of some intervals where the itineraries of the points are the same, the dynamics of the maps $f$ and $g$ are topologically equivalent(see [10]).

\subsection{The Lexicographical World}

Let $\operatorname{Min}_{2}=\left\{a \in \Sigma_{0} ; \sigma^{k}(a) \geq a, k \in N\right\}$ and $\operatorname{Max}_{2}=\left\{b \in \Sigma_{1} ; \sigma^{k}(b) \leq\right.$ $b, k \in N\}$.

Definition 2. The set $L W=\left\{(a, b) \in \operatorname{Min}_{2} \times \operatorname{Max}_{2} ;\{a, b\} \subset \Sigma_{a, b}\right\}$ will be called the lexicographical world.

For $a \in M i n_{2}$ its $L W$-fiber is the set $L W_{0}(a)=\left\{b \in \operatorname{Max}_{2} ;(a, b) \in\right.$ $L W\}$. For $b \in M a x_{2}$ its $L W$-fiber is the set $L W_{1}(b)=\left\{a \in \operatorname{Min}_{2} ;(a, b) \in\right.$ $L W\}$.

Remark 1. It is clear that given $(a, b) \in L W$ then $\Sigma_{a, b} \neq \emptyset$.

Let us now consider $(a, b) \in L W$.

Lemma 3. There is $f \in D M_{0}$ such that $I_{f}=\Sigma_{a, b}$. 
Proof: Let us consider the map $g:(R \backslash\{0\}) \rightarrow R$ given by

$$
g(x)= \begin{cases}2 x-1, & x>0 \\ 2 x+1, & x<0\end{cases}
$$

In this case $I_{g}=\Sigma_{2}$. Let $x_{a}<0$ and $x_{b}>0$ be the points such that $I_{g}\left(x_{a}^{+}\right)=a, I_{g}\left(x_{b}^{-}\right)=b$.

Let $f:(R \backslash\{0\}) \rightarrow R$ be the map defined by:

$$
f(x)=\left\{\begin{array}{lc}
g(x), & x \leq \overline{x_{b}} \\
x_{b}, & \overline{x_{b}} \leq x<0 \\
x_{a}, & 0<x \leq \overline{x_{a}} \\
g(x), & \overline{x_{a}} \leq x
\end{array}\right.
$$

here $\overline{x_{b}}<0<\overline{x_{a}}$ are given by $g\left(\overline{x_{b}}\right)=x_{b}$ and $g\left(\overline{x_{a}}\right)=x_{a}$.

We will call this result the realization lemma. It is clear that the maps, in this construction, are non-decreasing but we want a realization lemma with increasing maps. In section 3 we prove a realization lemma with increasing maps.

Therefore, we have a surjective map $I: D M_{0} \rightarrow L W, I(f)=\left(a_{f}, b_{f}\right)$ and $D M_{0}=\bigcup_{(a, b) \in L W} I^{-1}(\{(a, b)\})$. Also, using $C^{0}$ proximity on compact sets, this map is continuous.

In this context the next definition is natural.

Definition 3. Let $\alpha: U \subset R^{k} \rightarrow D M_{0}$ be a map.

1) We will say that $\alpha$ is an $a$-surjective family if $\forall a \in M i n_{2}$ there is a nonempty set , $A(a) \subset U$, such that $a_{f}=a, \forall f \in \alpha(A(a))$.

2) We will say that $\alpha$ is an $b$-surjective family if $\forall b \in M_{a x}$ there is a nonempty set, $B(b) \subset U$, such that $b_{f}=b, \forall f \in \alpha(B(b))$.

3) We will say that $\alpha$ is an $L W$-surjective family if $\forall(a, b) \in L W$ there

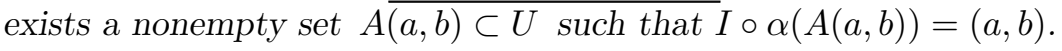

It is clear that associated to any map $\alpha$, as above, we have an $a(b, L W)$ decomposition of its domain. We will call this $a(\operatorname{resp} . b, L W)$-decomposition the $a$ (resp. $b, L W)$ bifurcation theory defined by $\alpha$.

Open Problem: Are there $L W$-surjective families?.

Certainly, this is a very hard problem and we believe that there is not a finite $k \in N$ with this property. In the present paper we prove the following 
Theorem 1. The given two parameter family of quadratic contracting Lorenz maps is a-surjective, is $b$-surjective but it is not $L W$-surjective. Moreover, the respective $a, b, L W$-bifurcation diagramme are given.

\section{Symbolic Dynamics and the Lexicographical World}

Here we introduce some results and notations that are necessary for our results. The results which we do not prove in this section are proved in $[21,22,23]$. In the sequel we will denote by $\underline{a}=a_{0}, a_{1}, \ldots a_{n}$ the periodic sequence $\left(a_{0}, a_{1}, \ldots, a_{n}, a_{0}, a_{1}, \ldots, a_{n}, \ldots\right) \in \Sigma_{2}$. Assume that we have a countable set of sequences, $A$. We will say that two elements $a<b \in A$ are consecutive in $A$ (or consecutive if the context is clear) if there does not exist an element $c \in A$ such that $a<c<b$

\subsection{Dynamical properties for sequences in LW}

Let $\underline{a_{1}}, \underline{a_{2}}$ be two periodic sequences in $\Sigma_{0}$. The periodic sequence $m\left(a_{1}, a_{2}\right)=$ $\underline{a_{1} a_{2}}$ will be called the average of the sequences $\underline{a_{1}}$ and $\underline{a_{2}}$.

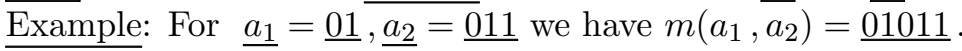

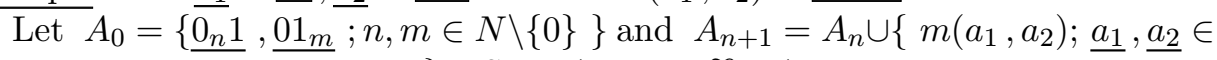
$A_{n}$ are consecutive sequences $\}$. Set $A_{\infty}=\bigcup_{n=0}^{\infty} A_{n}$. The elements in $A_{\infty}$ will be called primary sequences. As we will see in the next section, primary sequences are associated with primary bifurcations.

The elements in $\overline{A_{\infty}}$ are characterized by the following property:

Lemma 4. (see $[25,26]) a \in \overline{A_{\infty}}$ if and only if

$$
(*) \quad a \in \operatorname{Min}_{2} \text { and } \sigma(a) \geq \sigma(b),
$$

for $b=\sup \left\{\sigma^{k}(a) ; k \in N\right\}$.

Let us denote $A_{\infty}^{0}=A_{\infty}$ and define, for any $a \in A_{\infty}^{0}$ the set:

$$
A_{1}^{1}(a)=\left\{c \in \Sigma_{0} ; c=\underline{a_{-} b_{+} a^{n}} \text { or } c=\underline{a_{-} b^{m} b_{+}}, \text {for } n, m \in N\right\} .
$$

Here, for $b=b_{0} b_{1} \ldots b_{k} 0$ we denote $b_{+}=b_{0} b_{1} \ldots b_{k} 1$ and for $a=a_{0} a_{1} \ldots a_{k} 1$ we denote $a_{-}=a_{0} a_{1} \ldots a_{k} 0$.

Observe that if $c_{n}=\underline{a_{-} b_{+} a^{n}}$ then $c_{n} \rightarrow a_{-} b_{+} \underline{a}$ and if $d_{m}=\underline{a_{-} b^{m} b_{+}}$ then $d_{m} \rightarrow a_{-} \underline{b}$.

Set

$$
A_{2}^{1}(a)=\left\{m\left(a_{1}, a_{2}\right) ; a_{1}, a_{2} \in A_{1}^{1}(a) \text { are consecutive sequences }\right\} \cup A_{1}^{1}(a)
$$


and in general

$A_{n+1}^{1}(a)=\left\{m\left(a_{1}, a_{2}\right) ; a_{1}, a_{2} \in A_{n}^{1}(a)\right.$ are consecutive sequences $\} \cup A_{n}^{1}(a), n \geq 2$.

Let $A_{\infty}^{1}(a)=\bigcup_{n=1}^{n=\infty} A_{n}^{1}(a)$ and $A_{\infty}^{1}=A_{\infty}^{0} \cup \bigcup_{a \in A_{\infty}^{0}} A_{\infty}^{1}(a)$

At this stage we have to point out the following: Assume $a \in A_{\infty}^{0}$. Let $\tilde{a}=\sup \left\{\sigma^{k}(a) ; k \in N\right.$ and $\left.\sigma^{k}(a) \in \Sigma_{0}\right\}$ and $\tilde{b}=\inf \left\{\sigma^{k}(a) ; k \in\right.$ $N$ and $\left.\sigma^{k}(a) \in \Sigma_{1}\right\}$. Consider $\Sigma_{\tilde{a}, \tilde{b}}=\{\theta, N \rightarrow\{\tilde{a}, \tilde{b}\}\}$ be the set of sequences of the two symbols $\tilde{a}$ and $\tilde{b}$. Replace $0=\tilde{a}$ and $\tilde{b}=1$ and define $A_{0}(a)=\left\{\underline{0_{n} 1}, \underline{01_{m}} ; n, m \in N \backslash\{0\}\right\}$ and $A_{n+1}(a)=A_{n}(a) \cup$ $\left\{m\left(a_{1}, a_{2}\right) ; a_{1}, a_{2} \in A_{n}(a)\right.$ are consecutive sequences $\}$. Set $A_{\infty}(a)=\bigcup_{n=0}^{\infty} A_{n}(a)$ and $A_{\infty}^{\star}(a)=\left\{\inf \left\{\sigma^{k}(\alpha) ; k \in N\right\} ; \alpha=R_{\tilde{a}, \tilde{b}}(\eta) ; \eta \in A_{\infty}(a)\right\}$.

Lemma $2 \quad \bar{A}_{\infty}^{\star}(a)=\bar{A}_{\infty}^{1}(a)$.

As before, let $\sigma_{\tilde{a}, \tilde{b}}: \Sigma_{\tilde{a}, \tilde{b}} \rightarrow \Sigma_{\tilde{a}, \tilde{b}}$ be the shift map. We have

$\underline{\text { Lemma } 3} \alpha \in \overline{A_{\infty}^{\star}}(a)$ if and only if

$(\star) \alpha \in \operatorname{Min}_{2}(\tilde{a}, \tilde{b})$ and $\sigma_{\tilde{a}, \tilde{b}}(\alpha) \geq \sigma_{\tilde{a}, \tilde{b}}(\beta)$ for $\beta=\sup \left\{\sigma_{\tilde{a}, \tilde{b}}^{k}(\alpha) ; k \in N\right\}$

Inductively, for any $a \in A_{\infty}^{n}(a)$, let

$$
A_{1}^{n+1}(a)=\left\{c \in \Sigma_{0} ; c=\underline{a_{-} b_{+} a^{j}} \text { or } c=\underline{a_{-} b^{k} b_{+}} \text {for } j, k \in N\right\}
$$

Now, we define

$A_{2}^{n+1}(a)=\left\{m\left(a_{1}, a_{2}\right) ; a_{1}, a_{2} \in A_{1}^{n+1}(a)\right.$ are consecutive sequences $\} \bigcup A_{1}^{n+1}(a)$

and

$A_{m+1}^{n+1}(a)=\left\{m\left(a_{1}, a_{2}\right) ; a_{1}, a_{2} \in A_{m}^{n+1}(a)\right.$ are consecutive sequences $\} \bigcup A_{m}^{n+1}(a), m \geq 2$.

As before, define $A_{\infty}^{n+1}(a)=\bigcup_{m=1}^{\infty} A_{m}^{n+1}(a) ; A_{\infty}^{n+1}=\bigcup_{a \in A_{\infty}^{n}} A_{\infty}^{n+1}(a) \cup$ $A_{\infty}^{n}$ and finally, $A_{\infty}^{\infty}=\bigcup_{j=0}^{\infty} A_{\infty}^{j}$.

Note A similar construction; as we did in Lemma 2 and Lemma 3, for $a \in A_{\infty}^{\circ}$, we can do for any $a \in A_{\infty}^{\infty}$.

The elements in $\left(A_{\infty}^{\infty} \backslash A_{\infty}^{0}\right)$ will be called secondary sequences. As we will see in the next section secondary sequences are associated with secondary bifurcations.

Let denote by $B_{\infty}^{\infty}$ the set $\left\{\sup \left\{\sigma^{k}(a), k \in N\right\}, a \in A_{\infty}^{\infty}\right\}$. We will denote by $b(a)$ the sequence $\sup \left\{\sigma^{k}(a), k \in N\right\}$ for $a \in \Sigma_{0}$ and by $a(b)$, the 
sequence $\inf \left\{\sigma^{k}(b), k \in N\right\}$ for $b \in \Sigma_{1}$. Clearly, $b(a) \in \operatorname{Max}_{2}$ and $a(b) \in$ $\mathrm{Min}_{2}$.

It is clear that $\Sigma_{a, \underline{1}} \neq \phi$ for any $a \in \Sigma_{0}$. Hence we can define maps $\varphi, \psi, \chi: \Sigma_{0} \rightarrow \Sigma_{1}$ by :

$$
\begin{gathered}
\varphi(a)=\inf \left\{b \in \Sigma_{1} \mid \Sigma_{a, b} \neq \phi\right\} \\
\psi(a)=\inf \left\{b \in \Sigma_{1} ; \Sigma_{a, b} \text { contains } \infty \text {-elements }\right\}
\end{gathered}
$$

and

$$
\chi(a)=\inf \left\{b \in \Sigma_{1} ; \Sigma_{a, b} \text { is uncountable }\right\} .
$$

Clearly, $a_{1} \leq a_{2}$ imply $\varphi\left(a_{1}\right) \leq \varphi\left(a_{2}\right), \psi\left(a_{1}\right) \leq \psi\left(a_{2}\right)$ and $\chi\left(a_{1}\right) \leq$ $\chi\left(a_{2}\right)$ and for all $c \in \Sigma_{1}$ such that $c<\varphi(a)$ we have $\Sigma_{a, c}=\Phi$.

Examples : For any $0 \underline{01} \leq a \leq \underline{01}$ we have $\varphi(a)=\underline{10}, \psi(\underline{01})=\chi(\underline{01})=$ 11ㅁ. Also $\varphi(\underline{0})=\psi(\underline{0})=\chi(\underline{0})=1 \underline{0} ; \varphi(0 \underline{1})=\psi(0 \underline{1})=\underline{1} ; \varphi\left(\underline{0_{n} 1}\right)=$ $\underline{10_{n}}, \psi\left(\underline{0_{n} 1}\right)=\chi\left(\underline{0_{n} 1}\right)=1 \underline{10_{n}}$ and $\varphi\left(\underline{01_{m}}\right)=\underline{1_{m} 0}, \psi\left(\underline{01_{m}}\right)=\chi\left(\underline{01_{m}}\right)=$ $\underline{11_{m}} 0$.

\subsection{The Morse-Smale and the Entropy Zero cases}

Definition 4. a.- We will call a map $f \in D M_{0} \underline{\text { Morse-Smale }}$ if $a_{f} \in A_{\infty}^{0}$ and $b_{f}=\varphi\left(a_{f}\right)$.

b.- We will call a map $f \in D M_{0}$ essentially Morse-Smale if $a_{f} \in A_{\infty}^{\infty}$ and $b_{f}=\varphi\left(a_{f}\right)$.

We will denote by $M S_{0} \subset D M_{0}$ the set of Morse-Smale and essentially Morse-Smale maps . We call these maps Morse-Smale because its dynamics essentially reduces to a periodic orbit.

\section{Examples}

a.- Any $f \in D M_{0}$ such that $a_{f}=\underline{0^{n} 1}, b_{f}=\underline{10^{n}}$ or $a_{f}=\underline{01^{n}}, b_{f}=\underline{1^{n} 0}$ are Morse-Smale maps.

b.- Any $f \in D M_{0}$ such that $a_{f}=\underline{0011(01)^{n}}, b_{f}=\underline{11(01)^{n} 00}$ are essentially Morse-Smale maps.

Lemma 5. ([23]) Given $f \in D M_{0}$ be a "Morse-Smale" map we have that $\left[f\left(0^{+}\right), f\left(0^{-}\right)\right]=\bigcup_{i=0}^{p e r\left(a_{f}\right)-1} \overline{I_{i}}$, where $x \in I_{0}$ implies $I_{f}(x)=a_{f}$ and $\left.I_{f}\right|_{I_{j}}$ is constant and equal to $a_{j}=\sigma^{j}\left(a_{f}\right)$ for $0<j \leq \operatorname{per}\left(a_{f}\right)-1$. 
Lemma 6. Let $a \in A_{\infty}^{0}$. There is an injective $f \in D M_{0}$ such that $a_{f}=a$ and $b_{f}=\varphi(a)$.Moreover, this map satisfy $f\left(f\left(0^{+}\right)\right)=f\left(f\left(1^{-}\right)\right)$.

Proof: Let $a=a_{0}<a_{1}<\ldots<\left(a_{\text {last }}=a_{p}\right)<0 \underline{1}<1 \underline{0}<\left(b_{\text {first }}=\right.$ $\left.b_{p+1}\right)<b_{p+2}<\ldots<b_{k}=b(a)$, denote the consecutive elements in $\left\{\sigma^{j}(a) ; \quad j \in N\right\}$. Let $\ell<0<r$ and $I=[\ell, r]$. Consider closed intervals $L_{0}, L_{1}, \ldots, L_{k}$ such that $\bigcup_{i=0}^{k} L_{i}=[\ell, r] ; \operatorname{left}\left(L_{0}\right)=\ell, \operatorname{right}\left(L_{0}\right)=$ $\operatorname{left}\left(L_{1}\right), \ldots, \operatorname{right}\left(L_{p}\right)=0=\operatorname{left}\left(L_{p+1}\right), \ldots, \operatorname{right}\left(L_{k-1}\right)=\operatorname{left}\left(L_{k}\right) ; \operatorname{right}\left(L_{k}\right)=$ $r$. Here left $[x, y]=x$ and $\operatorname{right}[x, y]=y$.

Now, define an injective map $f$ that sends $L_{p+1} \rightarrow L_{0}, L_{p+2} \rightarrow L_{1}, \ldots, L_{k} \rightarrow$ $L_{k-p-1}$ and $L_{0} \rightarrow L_{k-p}, L_{1} \rightarrow L_{k-p+1}, \ldots, L_{p} \rightarrow L_{k}$. This map satisfy the lemma.

The next result follows immediately from this lemma:

Corollary 1. Let $a \in \overline{A_{\infty}^{0}} \backslash A_{\infty}^{0}$. There is an injective map $f \in D M_{0}$ such that $a_{f}=a$ and $b_{f}=\varphi(a) \square$.

We note that these maps can be considered as bijective maps on the circle.

Lemma 7. Let $a \in A_{\infty}^{\infty}$. There is $f \in D M_{0}$ an increasing map, such that $a_{f}=a$ and $\varphi(a)=b_{f}$.

Proof: Let

$$
a=a_{0}<a_{1}<\ldots<a_{p}<0 \underline{1}<1 \underline{0}<a_{p+1}<\ldots<a_{k} .
$$

denote the consecutive elements in $\left\{\sigma^{j}(a), j \in N\right\}$. Associated with $a_{s}, 0 \leq s \leq k$,consider closed intervals $L_{s}, s=0, \ldots k$, such that

$$
L_{0} \leq L_{1} \leq \ldots \leq L_{p} \leq L_{p+1} \leq \ldots \leq L_{k}
$$

$L_{i} \cap L_{i+1}=$ one point or $L_{i} \cap L_{i+1}=\emptyset$, otherwise.

Let $\eta:\{0,1, \ldots, k\} \rightarrow\{0,1, \ldots, k\}$ be the permutation defined by $\sigma\left(a_{j}\right)=a_{\eta(j)}$. Clearly, $\eta(p)=k$ and $\eta(p+1)=0$. Now, define an increasing map $f \in D M_{0}$ which satisfies $f\left(L_{i}\right)=L_{\eta(i)}$ and is increasing between $L_{i}$ and $L_{i+1}$ when $L_{i} \cap L_{i+1}=\emptyset \square$

In a similar way we obtain

Lemma 8. Associated to any $a \in \operatorname{Min}_{2}$ there is an increasing map $f \in$ $D M_{0}$ such that $a_{f}=a$ and $b_{f}=\varphi\left(a_{f}\right)$. 
In general we get

Lemma 9. For $a \in A_{\infty}^{\infty}$ and $d \in B_{\infty}^{\infty} \cap L W_{0}(a)$. There is an increasing map $f \in D M_{0}$ such that $a_{f}=a, b_{f}=d$.

As a consequence of these lemmas we have

Proposition 1. Given $(a, b) \in L W$ there is an increasing map $f \in D M_{0}$ such that $I(f)=\left(a_{f}, b_{f}\right)=(a, b)$.

This result is a generalization of a similar result obtained for expansive maps in [18].

Definition 5. We will call a map $f \in D M_{0}$ an entropy zero map if $b_{f} \leq$ $\chi\left(a_{f}\right)$.

One of the most interesting problems related with the bifurcation theory associated to a parameterized family of dynamical systems $\left\{f_{\lambda} ; \lambda \in U \subset\right.$ $\left.R^{k}\right\}$, is to describe the set $\left\{\lambda \in U ; f_{\lambda}\right.$ is an entropy zero map $\}$ (see for instance [30], [4] and the references there in ). For our quadratic family of Lorenz maps we will prove some results, in this direction, in section 4.6.

We observe that any Morse-Smale or essentially Morse-Smale map is an entropy zero map(see [23] where we characterized the entropy zero set in the Lexicographical World).

Remark We announce that in some forthcoming works, with Lautaro Vásquez and Solange Aranzubia, we will provide many of the proofs that here we left for the reader or were we quote it is easy to see among other news results.

\section{Proof of the Results.}

\subsection{The Contracting Family.}

Let us consider the two parameter family $F_{(\lambda, \mu)}:(R \backslash\{0\}) \rightarrow R, \mu \geq$ $0, \nu \geq 0$, of maps in $D M_{0}$

$$
F_{\mu, \nu}(x)=\left\{\begin{array}{c}
-\mu+x^{2}, x>0 \\
\nu-x^{2}, x<0
\end{array}\right.
$$

In this section we will provide the bifurcation theory associated to this family. 


\section{Injective Maps}

Let us define

$$
I M=\left\{(\mu, \nu) ;\left.\quad F_{(\mu, \nu)}\right|_{[-\mu, \nu]},[-\mu, \nu] \rightarrow[-\mu, \nu] \quad \text { is an injective map }\right\} .
$$

It is not hard to see that $I M=\left\{(\mu, \nu):(\mu-1 / 2)^{2}+(\nu-1 / 2)^{2} \leq \frac{1}{2}\right\}$.

We observe the following: let $(\mu, \nu) \in I M$ such that $\mu>0, \nu>0$. We have that $F_{(\mu, \nu)}(-\mu) \geq F_{(\mu, \nu)}(\nu)$ and, consequently, if $a(\mu, \nu)$ denotes the itinerary associated to $(-\mu)^{+}$and $b(\mu, \nu)$ denotes the itinerary associated to $(\nu)^{-}$then $a(\mu, \nu) \in \bar{A}_{\infty}^{\circ}$ and $b(\mu, \nu)=\sup \left\{\sigma^{k}(a(\mu, \nu)) ; k \in N\right\}$.

\subsection{Primary Bifurcations.}

We call the bifurcations in this section primary bifurcations because they appears as bifurcation curves for injective maps in $D M_{0}$ and can be studied by using rotation numbers( see for instance [6], [3], [13] and [17]).

\subsubsection{Fixed points:}

The fixed points of the map $F_{(\mu, \nu)}$ are given by

(a)

$$
\begin{aligned}
& y_{ \pm}(\nu)=\frac{-1 \pm \sqrt{1+4 \nu}}{2} \quad \text { for } \quad-1 / 4 \leq \nu \leq 0 \\
& y_{-}(\nu)=\frac{-1-\sqrt{1+4 \nu}}{2} \quad \text { for } \quad \nu \geq 0
\end{aligned}
$$

(b)

$$
\begin{array}{ll}
x_{ \pm}(\mu)=\frac{-1 \pm \sqrt{1+4 \mu}}{2} & \text { for } \quad-1 / 4 \leq \mu \leq 0 \\
x_{+}(\mu)=\frac{1+\sqrt{1+4 \mu}}{2} & \text { for } \quad \mu \geq 0
\end{array}
$$

We have $y_{-}(\nu) \leq y_{+}(\nu) \leq 0 \leq x_{-}(\mu) \leq x_{+}(\mu)$.

\subsubsection{Preimages of Zero}

(a) $\nu-x^{2}=0$ imply $x=-\sqrt{\nu}=y_{1}(\nu), \nu \geq 0$ 


$$
\begin{aligned}
& \nu-x^{2}=-\sqrt{\nu} \text { imply } x=-\sqrt{\nu+\sqrt{\nu}}=y_{2}(\nu) \\
& \nu-x^{2}=y_{n}(\nu) \text { imply } x=-\sqrt{\nu-y_{n}(\nu)}=y_{n+1}(\nu), n \geq 2 ;
\end{aligned}
$$

(b) $-\mu+x^{2}=0$ imply $x=\sqrt{\mu}=x_{1}(\mu), \mu \geq 0$

$$
\begin{aligned}
& -\mu+x^{2}=\sqrt{\mu} \text { imply } x=\sqrt{\mu+\sqrt{\mu}}=x_{2}(\mu) \\
& -\mu+x^{2}=y_{n}(\mu) \text { imply } x=\sqrt{\mu+x_{n}(\mu)}=x_{n+1}, n \geq 2 .
\end{aligned}
$$

\subsubsection{Curves of bifurcation (first generation)}

(a) The equation: $F\left(0^{-}\right)=$fixed point of the right hand side; is solved by:

$$
\nu=\frac{1 \pm \sqrt{1+4 \mu}}{2}
$$

(b) The equation: $F\left(0^{+}\right)=$fixed point of the left hand side; is solved by:

$$
-\mu=\frac{-1 \pm \sqrt{1+4 \nu}}{2}
$$

See the figure 1.

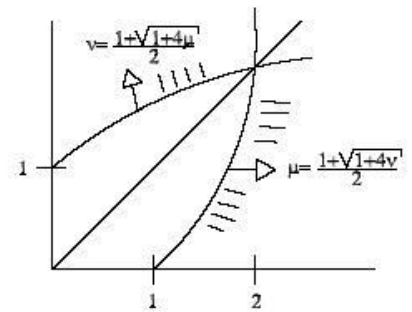

Figure 1:

We note (see figure 2 and 3 ) that:

$$
(\mu, \nu) \in R^{2} \text { such that } \nu \geq \frac{1+\sqrt{1+4 \mu}}{2}, \quad \mu \geq 0 \text { imply } b\left(F_{(\mu, \nu)}\right)=\underline{1}
$$


and

$(\mu, \nu) \in R^{2}$ such that $\mu \geq \frac{1+\sqrt{1+4 \nu}}{2}, \quad \nu \geq 0$ imply $a\left(F_{(\mu, \nu)}\right)=\underline{0}$

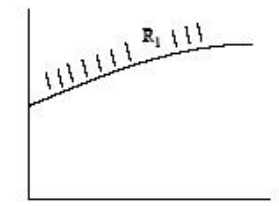

Figure 2:

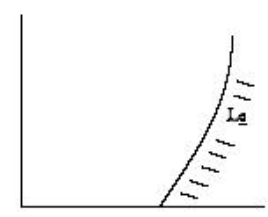

Figure 3:

Given $a \in \operatorname{Min}_{2}$ and $b \in \operatorname{Max}_{2}$ let denote by

$$
\begin{aligned}
& R_{b}=\left\{(\mu, \nu) \in R_{0}^{+} \times R_{0}^{+} ; \quad b\left(F_{(\mu, \nu)}\right)=b\right\} \\
& L_{a}=\left\{(\mu, \nu) \in R_{0}^{+} \times R_{0}^{+} ; \quad a\left(F_{(\mu, \nu)}\right)=a\right\}
\end{aligned}
$$

(c) $F\left(0^{+}\right)=n$-th preimage of zero $=y_{n}(\nu)$.

These equation define the curves $\mu_{n}(\nu)=-y_{n}(\nu)$ that satisfies:

$$
(\mu, \nu) \in\left\{\left(\mu_{n}(\nu) ; \nu\right) ; \quad \nu \geq 0\right\} \quad \text { imply } \quad a\left(F_{(\mu, \nu)}\right)=\underline{0_{n} 1} .
$$

These curves converges to the curve $(\mu(\nu), \nu)$ (see figure 4) where $\mu(\nu)$ is given by

$$
\mu(\nu)=\frac{1+\sqrt{1+4 \nu}}{2}, \nu \geq 0 .
$$




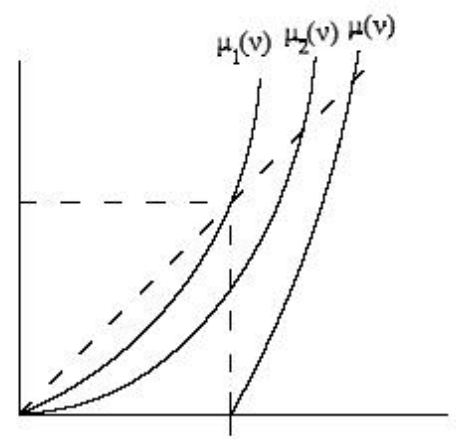

Figure 4:

In fact, we note that $\mu_{1}(\nu)=\sqrt{\nu}, \mu_{2}(\nu)=-y_{2}(\nu)=$ $\sqrt{\nu+\sqrt{\nu}}, \ldots, \mu_{n+1}(\nu)=-y_{n+1}(\nu)=\sqrt{\nu+\mu_{n}(\nu)}$.

Hence, taking the limit when $n \rightarrow \infty$, we get $\mu(\nu)=\sqrt{\nu+\mu(\nu)}$, so $\mu^{2}=\nu+\mu$ and, consequently, $\mu(\nu)=\frac{1+\sqrt{1+4 \nu}}{2}$ as announced.

We also observe that the curves $\left(\mu_{n}(\nu), \nu\right)$ transversally intersect the curve $\left(\mu, \frac{1+\sqrt{1+4 \mu}}{2}\right)$, any $n \in N$.

(d) $F\left(0^{-}\right)=n$-th preimage of zero $=x_{n}(\mu)$.

These equation define the curves $\nu_{n}(\mu)=x_{n}(\mu)$ that satisfies:

$$
(\mu, \nu) \in\left\{\left(\mu, \nu_{n}(\mu)\right) ; \quad \mu \geq 0\right\} \quad \text { imply } \quad b\left(F_{(\mu, \nu)}\right)=\underline{1_{n} 0 .}
$$

These curves converges to the curve $(\mu, s(\mu))$ (see figure 5 ) where $s(\mu)$ is given by

$$
s(\mu)=\frac{1+\sqrt{1+4 \mu}}{2}, \mu \geq 0 .
$$




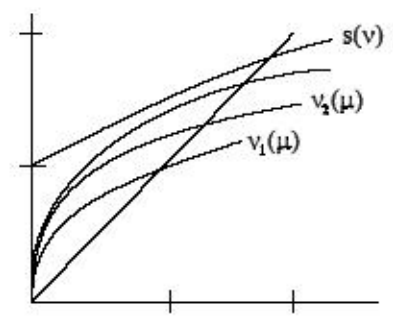

Figure 5:

Let us now prove the following

Proposition 2. The curve $C_{\underline{10}}=\left\{\left(\mu, \nu_{1}(\mu)\right), \mu \in\right] 0, \infty[\}$ transversally

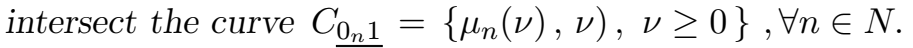

Proof: For $C_{1 \underline{10}}$ we have $C_{\underline{10}}=\{(\mu, \sqrt{\mu}) ; \mu>0\}$. Hence $C_{\underline{10}}$ is described by $\mu(\nu)=\nu^{2}, \nu>0$.

Now $C_{\underline{01}}$ is described as $\mu_{1}(\nu)=\sqrt{\nu}$. We have $C_{\underline{01}} \cap C_{\underline{10}}=\{(1,1)\}$. and $\mu_{1}^{\prime}(\nu)=\frac{1}{2 \sqrt{\nu}} ;\left(\nu^{2}\right)^{\prime}=2 \nu$. So $\mu_{1}(\nu)$ is transversal to $\mu(\nu)=\nu^{2}$ at $\nu=1$. Note $\mu_{1}^{\prime}(\nu) \leq \frac{1}{2}$ and $\mu^{\prime}(\nu) \geq 2$, all $\nu \geq 1=\nu_{1}$.

Inductively, assume that $C_{\underline{10}}$ transversally intersect $C_{\underline{0 n 1}}$ at a point $\left(\nu_{n}^{2}, \nu_{n}\right)$ with $\mu_{n}^{\prime}(\nu) \leq 1 / 2$ and $\overline{\mu^{\prime}}(\nu) \geq 2$, all $\nu \geq \nu_{n} \geq \nu_{n-1}$.

For the curve $C_{0_{n+1} 1}$ we have $\mu_{n+1}(\nu)=\sqrt{\nu+\mu_{n}(\nu)}$ and $\mu_{n+1}^{\prime}(\nu)=$ $\frac{1+\mu_{n}^{\prime}(\nu)}{2 \mu_{n+1}(\nu)}$

Let $\left(\nu_{n+1}^{2}, \nu_{n+1}\right) \in C_{0_{n+1} 1} \cap C_{\underline{10}}$. We have $\mu^{\prime}(\nu)=2 \nu \geq 2 \nu_{n+1}>$ $2 \nu_{n} \geq 2 \nu_{n-1} \geq \cdots \geq 2$ and $\mu_{n+1}^{\prime}(\nu)=\frac{1+\mu_{n}^{\prime}(\nu)}{2 \mu_{n+1}(\nu)} \leq \frac{3 / 2}{4}<1 / 2$, any $\nu \geq \nu_{n+1}>\nu_{n}$. So, we get the result.

Let us now prove

Proposition 3. The curve $C_{\underline{1_{m} 0}}=\left\{\left(\mu, \nu_{m}(\mu)\right) ; \mu \in\right] 0, \infty[\}$ transversally intersect the curve $C_{\underline{0_{n} 1}}, \forall n \overline{\in N}$.

Proof: For $m=2$ we have $\nu_{2}(\mu)=\sqrt{\mu+\sqrt{\mu}}$ then $\nu_{2}^{\prime}(\mu)=\frac{1+\frac{1}{2 \sqrt{\mu}}}{2 \nu_{2}(\mu)}=$ 
$\frac{1+\nu_{1}^{\prime}(\mu)}{2 \nu_{2}(\mu)}$

Since $\frac{1+\frac{1}{2 \sqrt{\mu}}}{2 \nu_{2}(\mu)}<1$ we get $\nu_{2}^{\prime}(\mu)<1$. Let denote by $\tilde{\mu_{2}}(\nu)$ its inverse map. We have $\tilde{\mu}_{2}^{\prime}(\nu)>1$. Since $\mu_{n}^{\prime}(\nu)<1$ we obtain the result in this case.

Inductively, assume $\nu_{m}^{\prime}(\mu)<1$ and that $\left\{\left(\mu, \nu_{m}(\mu)\right)\right\}$ transversally intersect the curve $C_{0_{n} 1}$.

Since $\nu_{m+1}^{\prime}(\mu)=\frac{\overline{1+} \nu_{m}^{\prime}(\mu)}{2 \nu_{m+1}(\mu)}<\frac{1+1}{2 \nu_{m+1}(\mu)}<1$, we get the result.

(e) Let us now assume that $F\left(0^{+}\right)<y_{1}(\nu)$.

In this situation there is $\overline{x_{1}}(\mu, \nu)$ such that $-\mu+{\overline{x_{1}}}^{2}=y_{1}(\nu)$; that is $\overline{x_{1}}(\mu, \nu)=\sqrt{\mu-\sqrt{\nu}}$.

If we ask for the condition $F\left(0^{-}\right)=\overline{x_{1}}(\mu, \nu)$ we get the curve $\overline{\mu_{1}}(\nu)=$ $\nu^{2}+\sqrt{\nu}$ (see figure 6). This curve is tangent to $\mu_{1}(\nu)$ at $\nu=0$ and transversally intersect $\mu_{n}(\nu), n \geq 2$ and $\mu(\nu)$.

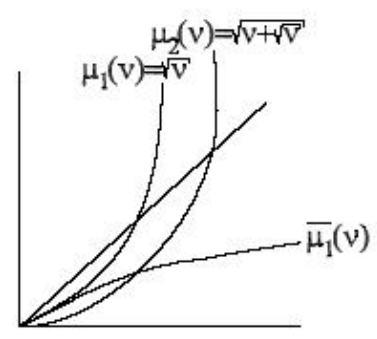

Figure 6:

In the same way: if we assume that $F\left(0^{+}\right)<y_{n}(\nu), n \geq 2$ we will find $\overline{x_{n}}(\mu, \nu)$ such that $-\mu+{\overline{x_{n}}}^{2}=y_{n}(\nu)$ that is $\overline{x_{n}}(\mu, \nu)=\sqrt{\mu+y_{n}(\nu)}$.

If we look for the condition $F\left(0^{-}\right)=\overline{x_{n}}(\mu, \nu)$ we get a curve $\overline{\mu_{n}}(\nu)=$ $\nu^{2}-y_{n}(\nu)$. This curve is tangent to $\mu_{n}(\nu)$ at $\nu=0$ and transversally intersect $\mu_{j}(\nu), j \geq n+1$.

We note that $(\mu, \nu) \in\left\{\left(\overline{\mu_{n}}(\nu), \nu\right) ; \quad \nu \geq 0\right\}$ imply $b\left(F_{(\mu, \nu)}\right)=\underline{10_{n+1}}$. 
(f) In a similar way if we assume $F\left(0^{-}\right)>x_{1}(\mu)$ we will find $\overline{y_{1}}(\mu, \nu)$ such that $\nu-{\overline{y_{1}}}^{2}=x_{1}(\mu)$ that is: $\overline{y_{1}}(\mu, \nu)=-\sqrt{\nu-x_{1}(\mu)}$.

The condition $F\left(0^{+}\right)=\overline{y_{1}}(\mu, \nu)$ imply the existence of a curve, $\overline{\nu_{1}}(\mu)$, such that $\overline{\nu_{1}}(\mu)=\mu^{2}+x_{1}(\mu)=\mu^{2}+\sqrt{\mu}, \mu \geq 0$. This curve (see figure 7 ) is tangent to $\nu_{1}(\mu)$ at $\nu=0$ and transversally intersect $\nu_{n}(\mu) ; n \geq 2$ and $\nu(\mu)$.

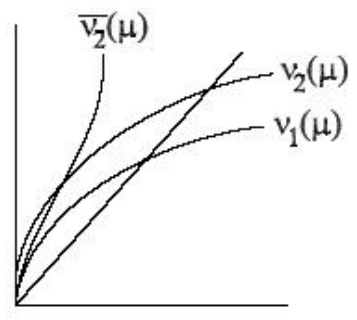

Figure 7:

In a similar way, if we assume that $F\left(0^{-}\right)>x_{n}(\mu), n \geq 2$ we will find $\overline{y_{n}}(\mu, \nu)$ such that $\nu-{\overline{y_{n}}}^{2}=x_{n}(\mu)$, that is $\overline{y_{n}}(\mu, \nu)=\sqrt{\nu-x_{n}(\mu)}$.

If we ask for the condition $F\left(0^{+}\right)=\overline{y_{n}}(\mu, \nu)$ we get the curve $\overline{\nu_{n}}(\mu)=$ $\mu^{2}+x_{n}(\mu), \mu \geq 0$. This curve is tangent to $\nu_{n}(\mu)$ at $\nu=0$ and transversally intersect $\nu_{j}(\mu) ; j \geq n+1$.

We note that $(\mu, \nu) \in\left\{\left(\mu, \overline{\nu_{n}}(\mu)\right) ; \quad \mu>0\right\}$ imply $a\left(F_{(\mu, \nu)}\right)=\underline{01_{n+1}}$.

Note : In this section we have proved :

1) That associated to any sequence $a \in A_{0}=\left\{\underline{0_{n} 1} ; \quad \underline{01_{n}} / n \in N\right\}$, we have two curves, $C(a)$ and $C(b(a))$, such that $(\mu, \nu) \in C(a)$ imply $a\left(F_{(\mu, \nu)}\right)=a$ and $(\mu, \nu) \in C(b(a))$ imply $b\left(F_{(\mu, \nu)}\right)=b(a)$.

Moreover, $C(a)$ and $C(b(a))$ transversally intersect at the point $\{P(a, b)\}=C(a) \cap C(b(a))$.

2) For $(\mu, \nu) \in C(a)$ we have that 0 is a fixed point of $F_{(\mu, \nu)}^{\# p e r(a)}$ that attracts a small right neighborhood of it.

3) For $(\mu, \nu) \in C(b(a))$ we observe that $x=\nu$ is a fixed point of $F_{(\mu, \nu)}^{\# p e r(b(a))}$ that attracts a small left neighborhood of it. 


\subsubsection{Transversality of the bifurcation curves.}

In this section we will prove that any curve of the form $C_{0_{n} 1_{p}}$ is transver-

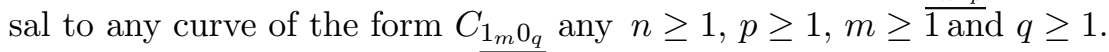

Assume that we have smooth maps. $\psi(\nu) ; \varphi(\mu)$ such that $G(\mu, \nu)=$ $-\mu+\psi(\nu)$ and $H(\mu, \nu)=\nu-\varphi(\mu)$ satisfy $G^{-1}(0)=C_{a} ; H^{-1}(0)=$ $C_{b} ; \psi^{\prime}(\nu) \leq 1, \nu \geq \nu_{0} ; \quad \varphi^{\prime}(\mu)<1, \mu \geq \mu_{0}$ and $C_{a} \cap C_{b}=\left\{\left(\mu_{0}, \nu_{0}\right)\right\}$

Lemma: Let $L(\mu, \nu)=-\mu+(G(\mu, \nu))^{2} ; M(\mu, \nu)=\nu-(H(\mu, \nu))^{2}$ then the curve $L^{-1}(0)$ is transversal to the curves $M^{-1}(0)$ and $H^{-1}(0)$. Moreover the curve $M^{-1}(0)$ is transversal to the curve $G^{-1}(0)$.

Proof: In fact.

$$
\begin{aligned}
& \frac{\frac{\partial L}{\partial \mu}}{\partial \nu}=-1+2 G(\mu, \nu) \cdot \frac{\partial G}{\partial \mu} \\
& \frac{\partial L}{\partial \nu}=2 G(\mu, \nu) \frac{\partial G}{\partial \mu} \\
& -\frac{\partial L}{\partial \mu}=-\frac{2 G(\mu, \nu) \frac{\partial G}{\partial \mu}-1}{2 G(\mu, \nu) \frac{\partial G}{\partial \nu}}=\frac{-\frac{\partial G}{\partial \mu}}{\frac{\partial G}{\partial \nu}}+\frac{1}{2 G(\mu, \nu) \frac{\partial G}{\partial \nu}}
\end{aligned}
$$

Since $\frac{\partial G}{\partial \mu}=-1, \frac{\partial G}{\partial \nu}=\psi^{\prime}(\nu)$ and for $L(\mu, \nu)=0$ we have $G(\mu, \nu)=\sqrt{\mu}$ then $\frac{-\frac{\partial L}{\partial \mu}}{\frac{\partial L}{\partial \nu}}=\frac{1}{\psi^{\prime}(\nu)}+\frac{1}{\left.2 \psi^{\prime} \nu\right) \cdot \sqrt{\mu}}=\frac{1}{\psi^{\prime}(\nu)}\left[1+\frac{1}{2 \sqrt{\mu}}\right]>1$

therefore $L^{-1}(0)$ is transversal to $H^{-1}(0)$ at any point $\left\{\left(\mu_{1}, \nu_{1}\right)\right\} \subset$ $L^{-1}(0) \cap H^{-1}(0)$ such that $\mu_{1} \geq \mu_{0}, \nu_{1} \geq \nu_{0}$.

For $M^{-1}(0)$ we have

$$
\begin{aligned}
\frac{-\frac{\partial M}{\partial \mu}}{\frac{\partial M}{\partial \nu}} & =\frac{2 H(\mu, \nu) \frac{\partial H}{\partial \mu}}{1-2 H(\mu, \nu) \frac{\partial H}{\partial \nu}}=\frac{2 \cdot(-\sqrt{\nu}) \cdot\left(-\varphi^{\prime}(\mu)\right)}{1-2(-\sqrt{\nu}) \cdot 1} \\
& =\frac{2 \varphi^{\prime}(\mu) \sqrt{\nu}}{1+2 \sqrt{\nu}}<1
\end{aligned}
$$

Hence $M^{-1}(0)$ is transversal to $L^{-1}(0)$.

A consequence of this Lemma is the following 
Corollary Let $\psi_{1}(\nu), \cdot, \psi_{n}(\nu) ; \varphi_{1}(\mu), \cdots, \varphi_{n}(\mu)$; be smooth maps such that $\psi_{i}^{\prime}(\nu)<1, \nu \geq \nu_{0} ; \varphi_{j}^{\prime}(\mu)<1, \mu \geq \mu_{0}$; and such that $G_{i}(\mu, \nu)=$ $-\mu+\psi_{i}(\nu)$ and

$H_{j}(\mu, \nu)=\nu-\varphi_{j}(\mu)$ satisfy $G_{i}^{-1}(0)=C_{a_{i}} ; H_{j}^{-1}(0)=C_{b_{j}}$ and $C_{a_{i}} \cap C_{b_{j}}=$ $\left\{\left(\mu_{j}, \nu_{i}\right)\right\}$ then for $L_{i}(\mu, \nu)=-\mu+\left(G_{i}(\mu, \nu)\right)^{2}, M_{j}(\mu, \nu)=\nu-\left(H_{j}(\mu, \nu)\right)^{2}$ we have that the curves $L_{i}^{-1}(0)$ are transversal to the curves $H_{j}^{-1}(0)$ and $M_{j}^{-1}(0)$. Moreover $M_{j}^{-1}(0)$ is traversal to $G_{j}^{-1}(0)$. since

This result apply to the curves $C_{0_{n} 1}=G_{n}^{-1}(0)$ and $H_{m}^{-1}(0)=C_{1_{m} 0}$

$$
\psi_{1}(\nu)=\sqrt{\nu}, \psi_{2}(\nu)=\sqrt{\nu+\psi_{1}(\nu)}, \cdots ; \psi_{n+1}(\nu)=\sqrt{\nu+\psi_{n}(\nu)} .
$$

and

$$
\varphi_{1}(\mu)=\sqrt{\mu}, \varphi_{2}(\mu)=\sqrt{\mu+\varphi_{1}(\mu)}, \cdots, \varphi_{n+1}(\mu)=\sqrt{\mu+\varphi_{n}(\mu)} .
$$

In particular we conclude that

(i) $C_{0_{n} 1}$ is transversal to $C_{1_{m} 0}$ for all $n \geq 1, m \geq 1$.

(ii) $C_{0_{n} 1}$ and $C_{0_{n} 11}$ are transversal to $C_{1_{m} 0}$ and, $C_{1_{m} 00}$ any $n \geq 1, m \geq 1$.

(iii) $C_{0_{n} 1}, C_{0_{n} 11}$ and,$C_{0_{1} 111}$ are transversal to $C_{1_{m} 0}, C_{1_{m} 00}$ and $C_{1_{m} 000}$ any $n \geq 1, m \geq 1$ and in general

(iv) $C_{0_{n} 1_{p}}$ is transversal to $C_{1_{m} 0_{q}}$ any $n \geq 1, p \geq 1, m \geq 1$ and $q \geq 1$ as we announced.

\subsubsection{Primary saddle-nodes.}

In this section we will prove

Proposition 4. Associated with any $a \in A_{0}$ and $b=b(a)$ there is a curve $S N(a, b) \subset R^{2}$ such that:

(i) there are values $\nu_{a, b}$ and $\mu_{a, b}$ such that $F_{\left(\mu_{a, b}, \nu_{a, b}\right)}^{\# p e r(a)}$ has a fixed point with derivative equal to 1 and $\left(\mu_{a, b}, \nu_{a, b}\right) \in S N(a, b)$;

(ii) $S N(a, b) \backslash\left\{\left(\mu_{a, b}, \nu_{a, b}\right)\right\}=S N(a) \cup S N(b)$ are two curves which satisfies: 
(a) $S N(a)$ transversally intersects $C(b)$ at a point $\left\{\left(\bar{\mu}_{a, b}, \bar{\nu}_{a, b}\right)\right\}=$ $S N(a) \cap C(b) . \quad S N(b)$ transversally intersects $C(a)$ at a point $\left\{\left(\tilde{\mu}_{a, b}, \tilde{\nu}_{a, b}\right)\right\}=S N(b) \cap C(a) ;$

(b) For any $(\mu, \nu) \in S N(a)$ there is a saddle-node periodic point which correspond to an unfolding of the fixed point of $F_{(\mu, \lambda)}^{\# p e r(a)}$, $(\mu, \nu) \in C(a)$.

(c) For any $(\mu, \nu) \in S N(b)$ there is a saddle-node periodic point which correspond to an unfolding of the fixed point of $F_{(\mu, \nu)}^{\# p e r(b)}$, $(\mu, \nu) \in C(b)$.

Proof: Initially, let us consider $a=\underline{01}$. In this situation the curve $C_{a}=$ $C_{01}=\left\{\left(\mu, \mu^{2}\right), \mu \geq 0\right\}$ represent the fact that $F\left(0^{+}\right)=y_{1}(\nu)$, hence $F\left(F\left(0^{+}\right)\right)=F\left(y_{1}(\nu)\right)=0$ that is, " $0^{+}$" is a periodic point whose period is \#(a). Also, for $b=\underline{10}$ the curve $C_{b}=C_{01}=\{(\mu, \sqrt{\mu}), \mu \geq 0\}$ represent the fact that $F\left(0^{-}\right)=x_{1}(\mu)$ and, then, $F\left(F\left(0^{-}\right)\right)=F\left(x_{1}(\mu)\right)=0$, that is " $0^{-}$" is a periodic point (of the map $F$ ) whose period is \#(b).

Let us consider the point $(1,1) \in C_{\underline{01}} \cap C_{\underline{10}}$. The graph of the map $F_{(1,1)}$ is as in left hand side of figure 8 and the graph of the restriction of the map $F_{(1,1)}^{2}$ is as in the right hand side of figure 8 (in the sequel, in this section, the restriction $\left.F_{(\mu, \nu)}^{2}\right|_{[0, \nu]}$ will be called the first return map to the interval $[o, \nu]$ or simply the first return map).
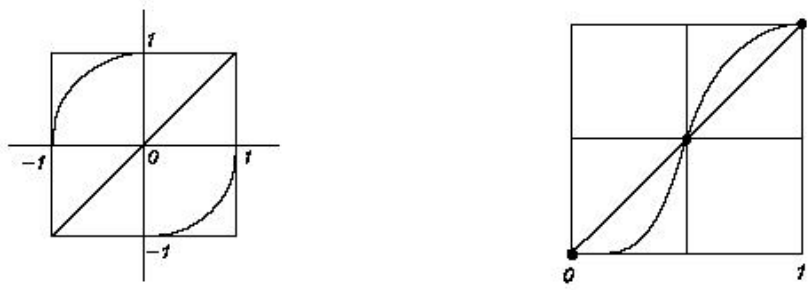

Figure 8:

Now, let $H=\{(\mu, \nu) ; \mu \geq 0, \nu \geq 0\}$. We have that $H \backslash C_{01}=$ $\left\{(\mu, \nu),, \mu^{2}<\nu\right\} \cup\left\{(\mu, \nu), \mu^{2}>\nu\right\}=A_{1} \cup A_{2}$ and $H \backslash C_{10}=\{(\mu, \nu), \sqrt{\mu}<$ $\nu\} \cup\{(\mu, \nu), \sqrt{\mu}>\nu\}=B_{1} \cup B_{2}$. So, we have the following components of the set $H \backslash\left(C_{a} \cup C_{b}\right)=A_{1} \cap B_{1} \cup A_{1} \cap B_{2} \cup A_{2} \cap B_{1} \cup A_{2} \cap B_{2}$ where $A_{1} \cap B_{1}=\left\{(\mu, \nu), \nu>\mu^{2}\right.$ and $\left.\nu>\sqrt{\mu}\right\}, A_{1} \cap B_{2}=\{(\mu, \nu), \sqrt{\mu}>\nu>$ $\left.\mu^{2}\right\}, A_{2} \cap B_{1}=\left\{(\mu, \nu), \mu^{2}>\nu>\sqrt{\mu}\right\}$ and $A_{2} \cap B_{2}=\left\{(\mu, \nu), \mu^{2}>\right.$ $\nu$ and $\sqrt{\mu}>\nu\}$. The next figures (see figures 9, 10 and 11) displays the 
first return map, to the interval $[0, \nu]$ for $(\mu, \nu) \in A_{i} \cap B_{j}, i=j=1,2$ and located near the point $(1,1)$.

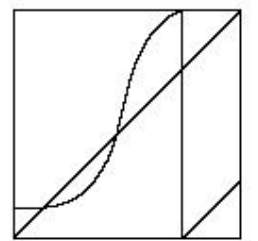

Figure 9: $A_{1} \cap B_{1}$

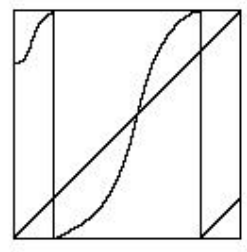

Figure 10: $A_{2} \cap B_{1}$

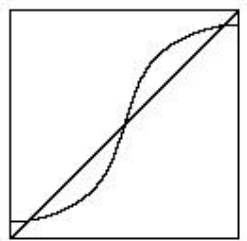

$A_{1} \cap B_{2}$.

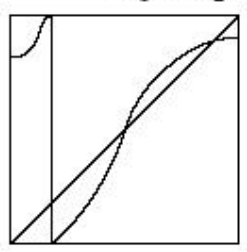

$A_{2} \cap B_{2}$.

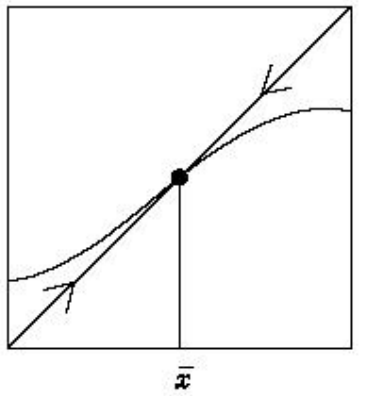

Figure 11:

Now, it is clear that we can find, in the interior of the set $A_{1} \cap B_{2}$, a point $\left(\mu_{a, b}, \nu_{a, b}\right)$ such that the first return map to the interval $[0, \nu]$ looks like in figure 11.

Figures 12 and 13 shown the bifurcation in the phase space, of the saddle node. 

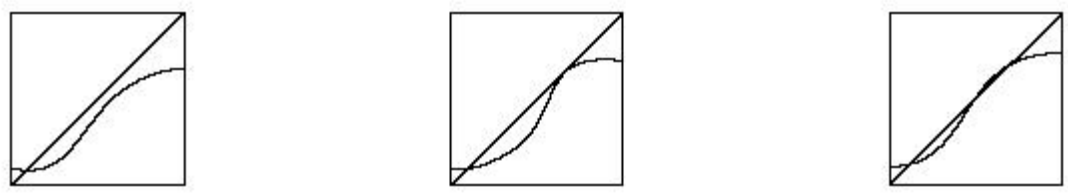

Figure 12:
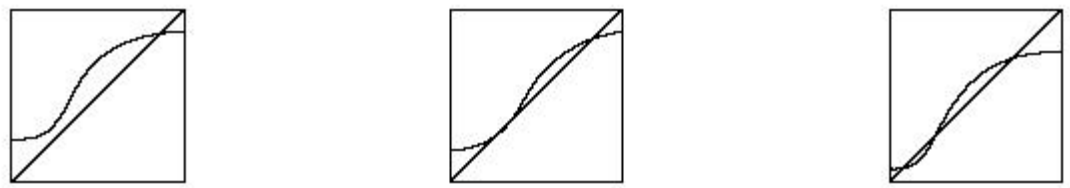

Figure 13:

Figures 14, 15 and 16 shown the essential facts of the curves $\operatorname{Sn}(a)$ and $S n(b)$ in the parameter plane.

Let us now consider the family $F_{\left(\mu, \mu^{2}+\rho\right)}, \rho \in R$. Clearly, there is a unique $\rho=\rho_{a, b}$ such that, for $\mu=\mu_{a, b}$ we have $\nu_{a, b}=\mu^{2}+\rho$. Now it is not hard to prove, for the one-parameter family $F_{\left(\mu, \mu^{2}+\rho\right)}, \rho$ fixed, the following

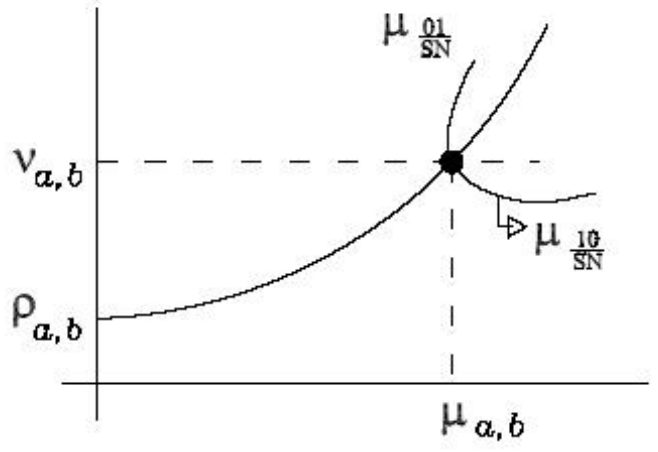

Figure 14: 


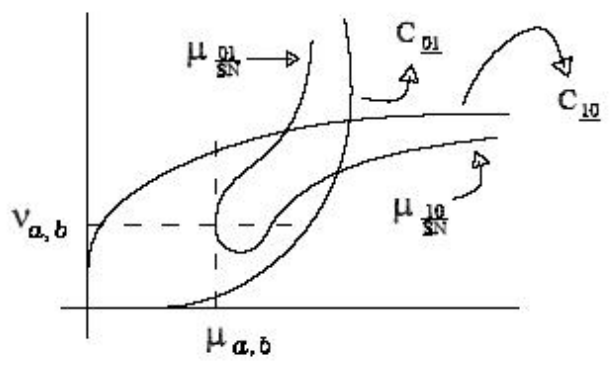

Figure 15:

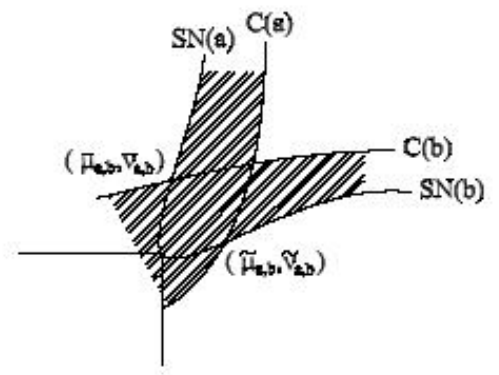

Figure 16:

Lemma 10. (i) Assume $\rho<\rho_{a, b}$ and near $\rho_{a, b}$. In this case the first return map has the generic unfolds showed in figure 12 :

(ii) Assume $\rho>\rho_{a, b}$ and near $\rho_{a, b}$. In this case the first return map has the generic unfold showed in figure 13 :

(iii) The curves $S N(b)=\mu \frac{10}{s n}(\rho)$ and $S N(a)=\mu \frac{01}{s n}(\rho)$ are given in figure 15,

(iv) The curve $S N(b)$ intersects the curve $\left\{\left(\mu, \mu^{2}+\rho\right), \mu \geq 0\right\}, \rho<\rho_{a, b}$ fixed, in a unique point.

(v) The curve $S N(a)$ intersects the curve $\left\{\left(\mu, \mu^{2}+\rho\right), \mu \geq 0\right\}, \rho>\rho_{a, b}$ fixed, in a unique point (see figure 16). 
This complete the proof in the case $a=\underline{01}$. The other cases follows in a similar way.

Figures 14, 15 and 16 displays the essential facts concerning the positions of the curves $S N(a) ; S N(b) ; C(a), C(b)$ and the region $L_{a}$ and $R_{b}$.

Remark 2. Given $a \in A_{0}, b=b(a)$ we define $L_{a}=\left\{(\mu, \nu) ; a\left(F_{(\mu, \nu)}\right)=a\right\}$ and $R_{b}=\left\{(\mu, \nu) ; \quad b\left(F_{(\mu, \nu)}\right)=b\right\}$.

It is not hard to prove that the global picture of the sets $L_{a}$ and $R_{b}$ looks like as in figure 17.
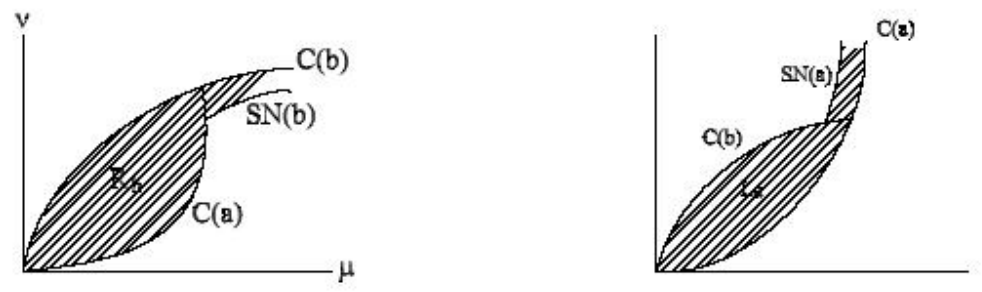

Figure 17:

\subsubsection{Bifurcation curves associated with averages}

Let us consider

$$
A_{1}=\left\{m\left(a_{1}, a_{2}\right) ; \quad a_{1}, a_{2} \in A_{0} \text { are consecutive sequences }\right\} \cup A_{0} \text {. }
$$

In a similar way as we did in section 4.2.4, we can prove that associated with $a=m\left(a_{1}, a_{2}\right), b=b(a)$ we can find two curves $C(a), C(b)$, tangent to $C\left(a_{2}\right)$ and $C\left(b\left(a_{2}\right)\right)$ respectively, such that:

$(\mu, \nu) \in C(a)$ imply $a\left(F_{(\mu, \lambda)}\right)=a$ and $(\mu, \nu) \in C(b)$ imply $b\left(F_{(\mu, \lambda)}\right)=b$.

Also, there are saddle-nodes associated to $C(a)$ and $C(b)$ as in 4.2.5. The geometry of the sets $R_{b}$ and $L_{a}$ is similar to the geometry of the sets described in the remark in 4.2.5. (see figure 18).

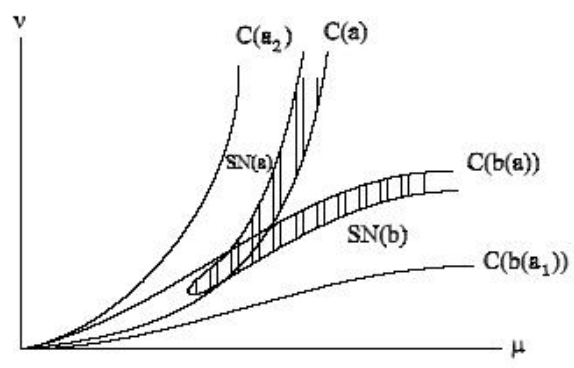

Figure 18: 

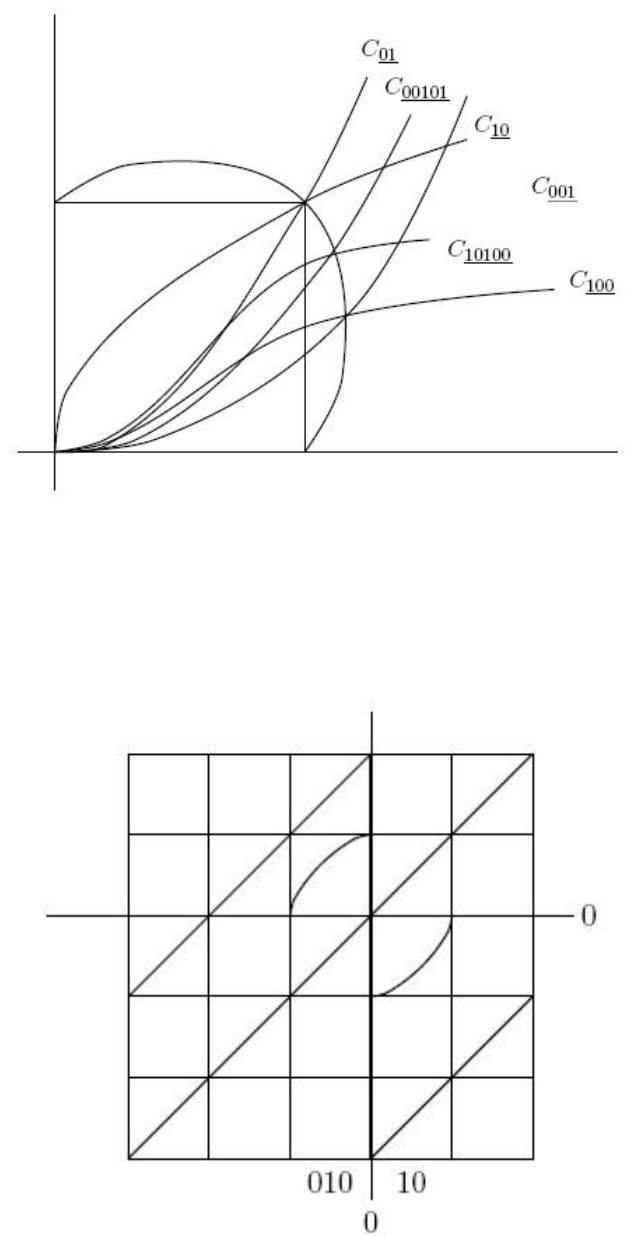

Similarly, we consider the set

$$
A_{n+1}=A_{n} \cup\left\{m\left(a_{1}, a_{2}\right) ; a_{1}<a_{2} \text { are consecutive sequences in } A_{n}\right\} .
$$

As before associated with $a \in A_{n+1}, a=m\left(a_{1}, a_{2}\right)$,we are able to find the curves $C(a), C(b(a))$ and $S N(a, b)$ (see figure 19).

In fact, without loss of generality, let us consider the sequences $a_{1}=\underline{01}$ and $a_{2}=\underline{001}$. 

$0)\}$

We have that $\left.C_{\underline{01}}=\{(\sqrt{\nu+\sqrt{\nu}}, \nu)) ; \nu>0\right\}$ and $C_{\underline{001}}=\{((\sqrt{\nu+\sqrt{\nu}}, \nu) \nu>$

Let us denote $\psi(\nu)=\sqrt{\nu+\sqrt{\nu}}, G(\mu, \nu)=-\mu+\psi(\nu), L(\mu, \nu)=-\mu+$ $(G(\mu, \nu))^{2}$ and $M(\mu, \nu)=\nu-(L(\mu, \nu))^{2}$. Now it is not hard to see that $M^{-1}(0)=C_{00101}$. In this situation $\left.L(\mu, \nu)\right)=\sqrt{\nu}$ and $G(\mu, \nu)=\sqrt{\mu-\sqrt{\nu}}$.

Moreover

$$
\begin{aligned}
\frac{-\frac{\partial M}{\partial \mu}}{\frac{\partial M}{\partial \nu}}= & -\frac{-2 L(\mu, \nu) \frac{\partial C}{\partial \mu}}{1-2 L(\mu ; \nu) \frac{\partial L}{\partial \nu}}= \\
= & \frac{-2 L(\mu, \nu)\left[-1+2 G(\mu, \nu) \cdot \frac{\partial G}{\partial \mu}\right]}{1-2 L(\mu, \nu) \cdot 2 G(\mu, \nu) \cdot \frac{\partial G}{\partial \nu}} \\
= & -\frac{2 \sqrt{\nu}[-1+2 \sqrt{\mu-\sqrt{\nu}} \cdot(-1)]}{1+4 \sqrt{\nu} \sqrt{\mu-\sqrt{\nu}} \psi^{\prime}(\nu)} \\
= & \frac{2 \sqrt{\nu}+4 \sqrt{\nu} \sqrt{\mu-\sqrt{\nu}}}{1+4 \sqrt{\nu} \sqrt{\mu-\sqrt{\nu}} \psi^{\prime}(\nu)}
\end{aligned}
$$

So,

$$
\frac{\frac{\partial M}{\partial \mu}}{\frac{\partial M}{\partial \nu}}>1
$$

if and any if $\quad(2 \sqrt{\nu}-1)+4 \sqrt{\nu(\mu-\sqrt{\nu})}\left(1-\psi^{\prime}(\nu)\right)>0$

Hence,

$$
-\frac{\frac{\partial M}{\partial \mu}}{\frac{\partial M}{\partial \nu}}>1 \quad \text { for } \quad \nu \geq 1 / 2
$$

Consequently $C_{10100}$ transversally intersects $C_{00101}$ since the intersection $C_{10100} \cap C_{00101}$ occurs for $\nu \geq 1 / 2, \mu \leq 1 / 2$.

For the curves involved we get the following picture 


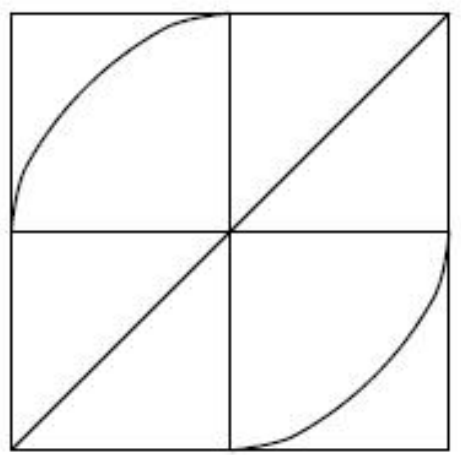

As we announced before.

With respect to the saddle note bifurcation let us consider the graph of the map $C_{00101} \cap C_{10100}$. The next figure displays the graph of the map $F_{(\mu, \nu)}$ such that $\{(\mu, \nu)\}=C_{\underline{00101}} \cap C_{\underline{10100}}$.

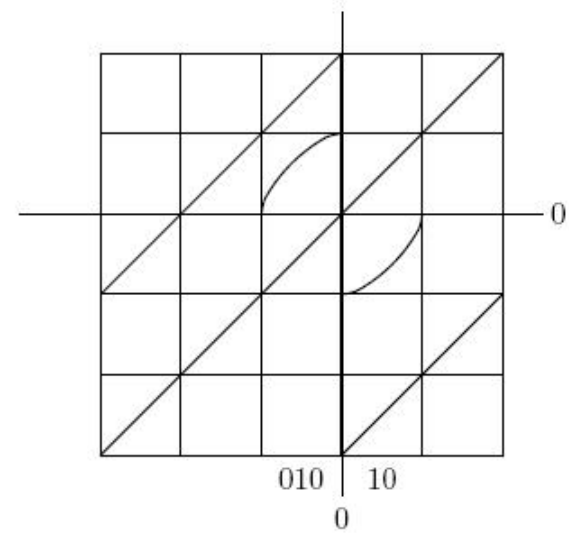

Now, it not hard to see that the first return map $\bar{F}: I_{010} \cup I_{10} \rightarrow$ $I_{010} \bigcup I_{10}$ has a graph as in the previous figure. Applying the results proved at the section 4.25 , to this return map, we get the announced saddlenode. Here $I_{a_{0}, \cdots, a_{p}}$ denotes the interval $\left\{x \in[-\mu, \nu] ; I_{0}(x)=a_{0}, I_{1}(x)=\right.$ $\left.a_{1}, \cdots, I_{p}(x)=a_{p}\right\}$.

4.2.7 Let $A_{\infty}=\bigcup_{n=0}^{\infty} A_{n}$. Assume $a \in \overline{A_{\infty}}$ is a sequence which is not periodic nor eventually periodic. Let $b=\sup \left\{\sigma^{k}(a) ; \quad k \in N\right\}$ and $a_{n} \in$ 
$A_{\infty}, a_{n} \rightarrow a$. let $b_{n}=b\left(a_{n}\right)$. It is clear that we have $b_{n} \rightarrow b$.

In this situation we can find values of the parameter $\left(\mu_{a}, \nu_{a}\right)$ and curves $C(a), C(b)$ such that: $C(a)=\left\{(\mu, \nu) ; \quad \nu=\varphi_{a}(\mu)\right\} ; C(b)=\{(\mu, \nu) ; \quad \nu=$ $\psi_{b}(\mu)$ where $\varphi_{a}:(0, \infty) \rightarrow R, \psi_{b}:(0, \infty) \rightarrow R$ satisfies

- $\varphi_{a}(\mu)=\psi_{b}(\mu), 0<\mu \leq \mu_{a}$.

- The maps $\left.\varphi_{a}\right|_{\left(\mu_{a}, \infty\right)}$ and $\left.\varphi_{b}\right|_{\left(\mu_{a}, \infty\right)}$ are tangent at $\mu_{a}$.

Moreover

(i) the curves $C\left(a_{n}\right)$ converges to $C(a)$ and the curves $C\left(b_{n}\right)$ converges to $C(b)$ (see figure 19) ;

(ii) the intersections $C\left(a_{n}\right) \cap C\left(b_{n}\right)$ converges to $\left\{\left(\mu_{a}, \nu_{a}\right)\right\}$;

(iii) $(\mu, \nu) \in C(a)$ imply $a\left(F_{(\mu, \lambda)}\right)=a$ and $(\mu, \nu) \in C(b) \operatorname{imply} b\left(F_{(\mu, \lambda)}\right)=b$.

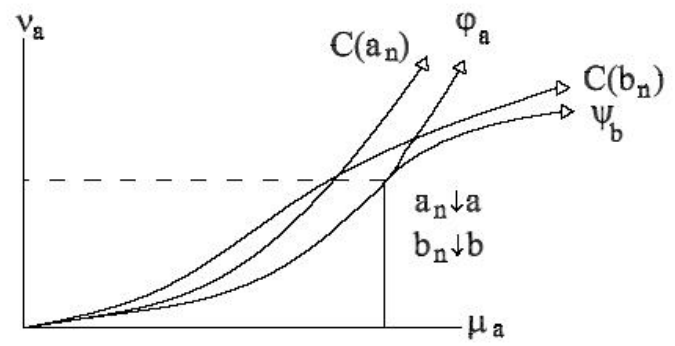

Figure 19:

Maybe, the unique point which is not easy to agree in this result is related with the coincidence of the curves $C(a)$ and $C(b)$ along $\left\{\left(\mu, \varphi_{a}(\mu)\right)\right.$; $\left.0<\mu \leq \mu_{a}\right\}$, but this is an easy consequence of the next result.

Let $r>0$ be a small number. Let $B_{r}^{+}=\left\{(\mu, \nu) \in R^{2}, \sqrt{\mu^{2}+\nu^{2}} \leq r\right.$, $\mu \geq 0, \quad \nu \geq 0\}$. Let $m(A)$ denotes the Lebesgue measure of the set $A \subset R^{2}$.

Lemma 11. $m\left(\left\{(\mu, \nu) \in B_{r}^{+} ; \quad F_{(\mu, \nu)} \notin M S_{0}\right\}\right)=0$.

Proof: In fact, the proof can be done in a similar way as was done in [19] or in [34].

Remark 3. 1) In fact, Arnold ([3] and Boyland ( [6]), obtained a similar result for the structure of the irrational Arnold's Tongues of the canonical family $f_{\lambda, b}(x)=x+\lambda+\frac{b}{2 \pi} \sin (2 \pi x)$.

2) A similar result(as in lemma 13) was proved by Swiatek in [38] with respect to parameterized families of homeomorphisms of the circle. 


\subsection{Secondary Bifurcations}

We call the bifurcations in this section secondary because this kind of changes in the dynamic cannot appear for injective maps in $D M_{0}$. Also, it is not possible to understand this scenario only by using rotation numbers as it is possible to see by comparing our results with those of [6].

The main idea on what follows is the following: Let $a \in A_{\infty}, b=$ $b(a)$ and $C_{a} \cap C_{b}=\left\{\left(\mu_{0}, \nu_{0}\right)\right\}$. Let $\tilde{a}=\sup \left\{\sigma^{k}(a) ; \sigma^{k}(a) \in \sum_{0}\right\}, \tilde{b}=$ inf $\left\{\sigma^{k}(b) ; \sigma^{k}(b) \in \sum_{1}\right\}$.

Associated with $\tilde{a}$ and $\tilde{b}$ we can find intervals

$$
I_{\tilde{a}}, I_{\tilde{b}} ; I_{\tilde{a}}=[\tilde{a}, 0], I_{\tilde{b}}=[0, \tilde{b}]
$$

such that the first return map $\tilde{F}_{\left(\mu_{0}, \nu_{0}\right)}=\left.F_{\left(\mu_{0}, \nu_{0}\right)}^{s}\right|_{I \tilde{a} \cup I \tilde{b}}: I_{\tilde{a}} \cup I_{\tilde{b}} \rightarrow I_{\tilde{a}} \cup I_{\tilde{b}}$ looks like in the following picture.
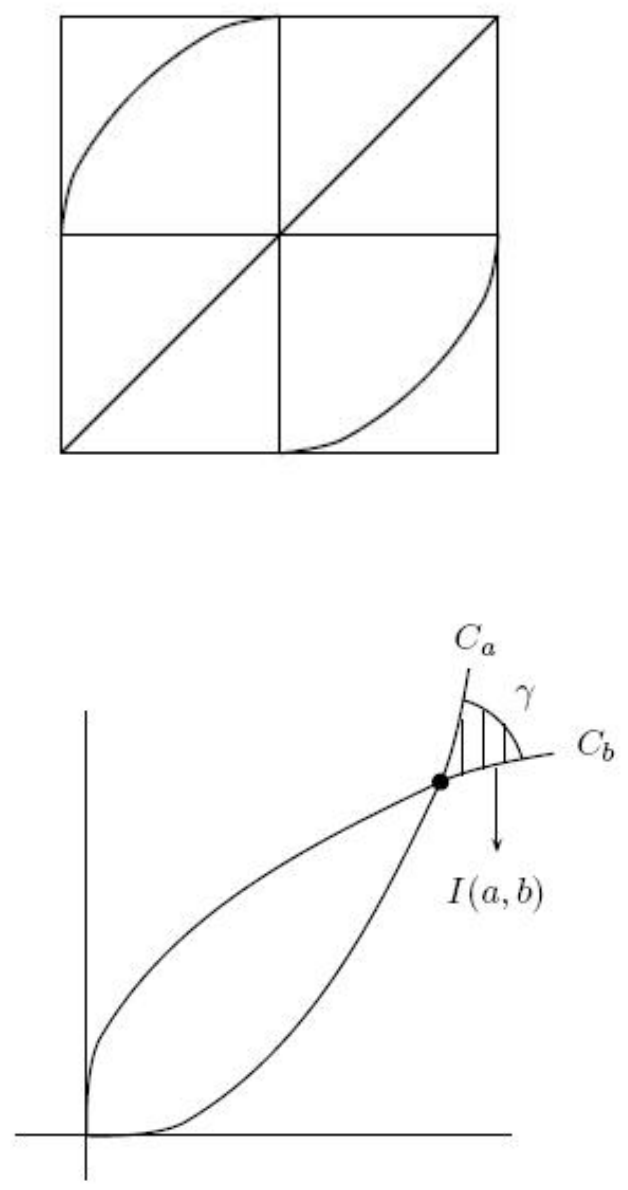
As in the case for elements in $A_{0}$, we can find, in set $C_{a b}=\{(\mu, \nu) ; \mu \geq$ $\left.\psi_{a}(\nu) ; \nu \geq \varphi_{b}(\mu)\right\}$, an injectivity region $I(a, b)$ for the map $\tilde{F}_{(\mu, \nu)}$. As before we can find a curve, $\gamma \subset C_{a, b}$, which define the boundary of the injectivity region(see next figure). Now we can proceed as before using $0=\tilde{a}, 1=\tilde{b}$ as the new symbols to obtain the previous constructions. That is, we will work with a renormalization map $\tilde{F}_{(\mu, \nu)}$; to obtain the results which we announce in the section below.

4.3.1 Let $B_{\infty}=\left\{b(a) ; \quad a \in A_{\infty}\right\}$. Let us now consider $a \in A_{\infty}, b \in B_{\infty}$ such that $C(a) \cap C(b) \neq \emptyset$.It is not hard to prove that ( see section 4.2.3) that:

a) $C_{a} \cap C_{b}=\{P(a, b)\}$ is a unique point.

b) define $A(a, b)=\left\{c \in \Sigma_{0} ; \quad c=\underline{a_{-} b_{+} a^{n}} ; n \in N\right.$ or $c=\underline{a_{-} b^{m} b_{+}} ; m \in$ $N\}$ and

$B(a, b)=\left\{d \in \Sigma_{1} ; d=\underline{b_{+} a^{n} a_{-}}, \quad n \in N\right.$ or $\left.d=\underline{b_{+} a_{-} b^{m}} ; m \in N\right\}$.

((i) Associated with $c=a_{-} b_{+}$there is a curve $C_{c}$, tangent to $C_{b}$; $P(a, b) \in \overline{C_{c}}$ and such that $(\mu, \nu) \in C_{c}$ imply $a\left(F_{(\mu, \nu)}\right)=c$ (see figure 20);

((ii) Associated with $d=b_{+} a$ there is a curve $C_{d}$, tangent to $C_{a}$; $P(a, b) \in \overline{C_{d}}$ and such that $(\mu, \nu) \in C_{d}$ imply $b\left(F_{(\mu, \nu)}\right)=d$ (see figure 20);

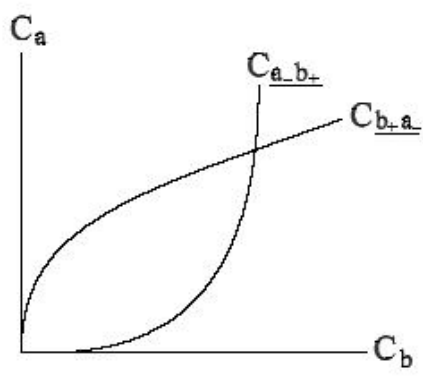

Figure 20: (b)(i) and (b)(ii) 
(iii) Associated with $c=a_{-} b_{+} a^{n}, n \geq 1$, there is a curve $C_{c}$, tangent to $C_{a} ; P(a, b) \in \overline{C_{c}}$ and such that $(\mu, \nu) \in C_{c}$ imply $a\left(F_{(\mu, \nu)}\right)=c$ (see figure 21);

(iv) Associated with $d=b_{+} a^{n} a_{-}, n \geq 1$, there is a curve $C_{d}$, tangent to $C_{a} ; P(a, b) \in \overline{C_{c}}$ and such that $(\mu, \nu) \in C_{d}$ imply $b\left(F_{(\mu, \lambda)}\right)=d$ (see figure 21 );

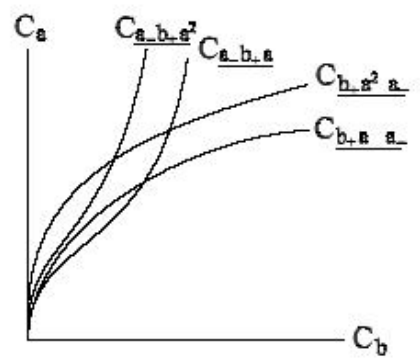

Figure 21: (b)(iii) and (b)(iv)

(v) Associated with $c=a_{-} b^{n} b_{+}, n \geq 1$, there is a curve $C_{c}$, tangent to $C_{b} ; P(a, b) \in \overline{C_{c}}$ and such that $(\mu, \lambda) \in C_{c}$ imply $a\left(F_{(\mu, \lambda)}\right)=c$ (see figure 22);

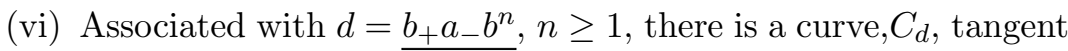
to $C_{b} ; P(a, b) \in \overline{C_{d}}$ and such that $(\mu, \lambda) \in C_{d}$ imply $b\left(F_{(\mu, \lambda)}\right)=d$ (see figure 22);

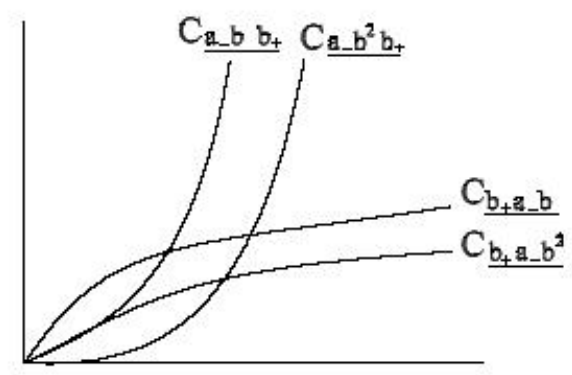

Figure 22: (b)(v) and (b)(vi)

In fact, let denote by $\xi_{b}:\left[\mu_{b}, \infty\left[\rightarrow R\right.\right.$ and $\xi_{a}:\left[\mu_{a}, \infty[\rightarrow R\right.$ the maps that satisfies: 
(i) $\left\{\left(\mu ; \xi_{b}(\mu)\right\} ; \quad \mu \geq \mu_{b}\right\}$ is the curve which represents the fact that $x=v$ is a periodic point with itinerary $b$;

(ii) $\left\{\left(\mu ; \xi_{a}(\mu)\right\} ; \quad \mu>\mu_{a}\right\}$ is the curve which represents the fact that $x=-\mu$ is a periodic point with itinerary $a$.

Let denote $C_{a} \cap C_{b}=\left\{\left(\overline{\mu_{b}}, \overline{\nu_{b}}\right)\right\}$.

Now, associated with $c_{n}=b_{+} a^{n} a_{-}, n \geq 0$ we can obtain maps $\xi_{n}$ : $\left[\overline{\mu_{b}}, \infty[\rightarrow R\right.$ such that

(i) $\xi_{n}^{\prime}\left(\overline{\mu_{b}}\right)=\xi_{a}^{\prime}\left(\overline{\mu_{b}}\right)-\xi_{b}^{\prime}\left(\mu_{b}\right) ; \xi_{n}\left(\overline{\mu_{b}}\right)=0$

(ii) $\left\{\left(\mu ; \xi_{b}(\mu)+\xi_{n}(\mu)\right) ; \quad \mu \geq \overline{\mu_{b}}\right\}$ is the curve which represents the fact that $x=v$ is a periodic point with itinerary $c_{n}$.

In a similar way, associated with $d_{m}=b_{+} a_{-} b^{m}, m \geq 1$, there are maps $\phi_{m}:\left[\overline{\mu_{b}}, \infty[\rightarrow R\right.$ such that

(i) $\phi_{m}\left(\overline{\mu_{b}}\right)=0 ; \phi_{m}\left(\overline{\mu_{b}}\right)=0$.

(ii) $\left\{\left(\mu, \xi_{b}(\mu)+\phi_{m}(\mu)\right) ; \quad \mu \geq \overline{\mu_{b}}\right\}$ is the curve which represents the fact that $x=v$ is a periodic point with itinerary $d_{m}$.

(c) Associated with $c=a_{-} \underline{b}$ there is a curve $C_{c}$, tangent to $S N(b)$ at the point $P_{c} \in C_{c}$, transversal to $C_{b}$ at the point $Q_{c}$, such that $(\mu, \lambda) \in C_{c}$ imply $a\left(F_{(\mu, \lambda)}\right)=c$ (see figure 23 ).

Moreover let $L_{c}$ be the open rectangle bounded by $C_{a}, S N(b), C_{c}$ and $C_{b}$. We have $L_{c} \subset R_{b}$ and $(\mu, \nu) \in L_{c}$ imply $a\left(F_{(\mu, \lambda)}\right)=c$. Also, $(\mu, \lambda) \in \overline{L_{c}} \cap\left[C_{b} \cup S N(b) \backslash C_{a}\right]$ imply $a\left(F_{(\mu, \lambda)}\right)=c$;

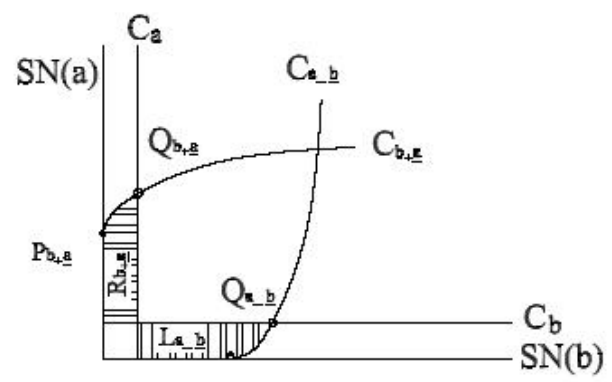

Figure 23: (c) and (e)

(d) Associated with $d=b_{+} a_{-} \underline{b}$ there is a curve $C_{d}$, tangent to $C_{a_{-} \underline{b}}$ at the point $Q_{a_{-} \underline{b}} \in \overline{C_{d}} \cap C_{a_{-} \underline{b}}$ and such that $(\mu, \nu) \in C_{d}$ imply $b\left(F_{(\mu, \lambda)}\right)=d$ 
(see figure 24);

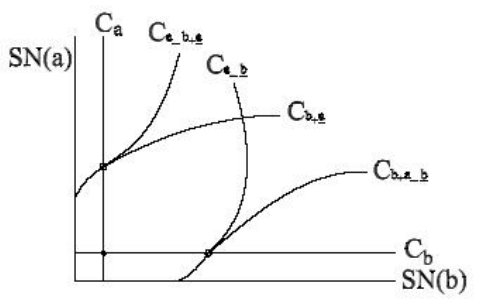

Figure 24: (d) and (f)

(e) Associated with $d=b_{+} \underline{a}$ there is a curve, $C_{d}$, tangent to $S N(a)$ at the point $P_{d} \in C_{d}$, transversal to $C_{a}$ at the point $Q_{d}$ such that $(\mu, \nu) \in C_{d}$ imply $b\left(F_{(\mu, \lambda)}\right)=d$ (see figure 23$)$. Moreover, let $R_{d}$ be the open rectangle bounded by $C_{a}, S N(a), C_{b}$ and $C_{d}$. We have $R_{d} \subset L_{a}$ and $(\mu, \nu) \in R_{d}$ imply $b\left(F_{(\mu, \lambda)}\right)=d$. Also,

$$
(\mu, \lambda) \in \overline{R_{d}} \cap\left[C_{a} \cup S N(a) \backslash C_{b}\right] \text { imply } b\left(F_{(\mu, \lambda)}\right)=d ;
$$

(f) Associated with $c=a_{-} b_{+} \underline{a}$ there is a curve $C_{c}$, tangent to $C_{b_{+} \underline{a}}$ at the point $Q_{b_{+} \underline{a}} \in \overline{C_{c}} \cap C_{b_{+} \underline{a}}$, and such that $(\mu, \nu) \in C_{c}$ imply $a\left(F_{(\mu, \lambda)}\right)=c$.

Figures (20),(21),(22),(23) and (24) displays the properties in 4.3.1.

Remark 4. (i) Let $\gamma\left(a, a_{-} b_{+} \underline{a}\right)$ be the curve which start in $P(a, b)$, follows $C_{a}$ up to $Q_{b_{+} \underline{a}}$ and then follows $C_{a_{-} b_{+} \underline{a}}$. We have that the sequence of curves

$$
\left\{C_{a_{-} b_{+} a^{m}} ; m \in N\right\} \quad \text { converges to } \quad \gamma\left(a, a_{-} b_{+} \underline{a}\right) ;
$$

(ii) Let $\gamma\left(a, b_{+} \underline{a}\right)$ be the curve which start in $P(a, b)$, follows $C_{a}$ up to $Q_{b_{+} \underline{a}}$ and then follows $C_{b_{+} \underline{a}}$. We have that the sequence of curves $\left\{C_{b_{+} a^{m} a_{-}}, m \in N\right\}$ converges to $\gamma\left(a, b_{+} \underline{a}\right)$;

(iii) Let $\gamma\left(b, a_{-} \underline{b}\right)$ be the curve which start in $P(a, b)$, follows $C_{b}$ up to $Q_{a_{-} \underline{b}}$ and then follows $C_{a_{-} b}$. We have that the sequence of curves $\left\{C_{a_{-} b^{m} b_{+}} ; m \in N\right\}$ converges to $\gamma\left(b, a_{-} \underline{b}\right)$;

(iv) Let $\gamma\left(b, b_{+} a_{-} \underline{b}\right)$ be the curve which start in $P(a, b)$, follows $C_{b}$ up to $Q_{a_{+} \underline{b}}$ and then follows $C_{b_{+} a_{-} \underline{b}}$. We have that the sequence of curves $\left\{C_{b_{+} a_{-} b^{m}}, m \in N\right\}$ converges to $\gamma\left(b, b_{+} a_{-} \underline{b}\right)$;

(v) The same construction that we did here can be done for any $a \in A_{\infty}^{\infty}$, $b \in B_{\infty}^{\infty}$ such that $C_{a} \cap C_{b} \neq \emptyset$.

4.3.2 We will say that $f \in D M_{0}$ satisfy the axiom $A$ if: 
(i) All the attracting periodic point of $f$ are hyperbolic and

(ii) The complement of the stable manifolds of the attracting periodic orbits, in $\left[f\left(0^{+}\right), f\left(0^{-}\right)\right]$, is an hyperbolic set.

Lemma 12. Let $a \in \mathcal{A}_{\infty}^{\infty}$ and $b \in \beta_{\infty}^{\infty}$ such that $C_{a} \cap C_{b} \neq \emptyset$. There is a neighborhood $U(a, b) \subset R^{2}$ of the point $P(a, b)$ such that

$$
m\left(\left\{(\mu, \lambda) \in U(a, b) ; F_{(\mu, \nu)} \text { do not satisfy axiom } A\right\}\right)=0 .
$$

That is; the measure of the bifurcation in $U(a, b)$ in zero.

Proof This result follows as similar results in [19] or [34].

\subsection{Doubling Periods}

Let us consider $a \in A_{\infty}^{\infty}$ and $b \in \beta_{\infty}^{\infty}$ such that $C_{a} \cap C_{b} \neq \emptyset$.

We will use $a_{0}=a, b_{0}=b$. Let us construct the following sequences:

$$
a_{n+1}=\underline{\left(a_{n}\right)_{-}\left(b_{n}\right)_{+}} ; \text {and } b_{n+1}=\underline{\left(b_{n}\right)_{+}\left(a_{n}\right)_{-}} .
$$

We have

$$
\begin{aligned}
& a_{0}>a_{1}>a_{2}>\ldots>\lim _{n \rightarrow \infty} a_{n}=c \\
& b_{0}<b_{1}<b_{2}<\ldots<\lim _{n \rightarrow \infty} b_{n}=d
\end{aligned}
$$

We will call the sequence $\left(a_{n}, b_{n}\right)$ the doubling period sequence with starting point $\left(a_{0}, b_{0}\right)$. The pair $(c, d)$ will be called the target of the doubling period with starting point $\left(a_{0}, b_{0}\right)$.

Lemma 13. Associated with $c($ resp. $d)$ there is a curve $C_{c}\left(\right.$ resp. $\left.C_{d}\right)$ which is the limit of the curves $C_{a_{n}}$ (resp. $C_{b_{n}}$ ). Moreover $C_{c} \cap C_{d}$ contains a unique point $P(c, d)$.

For $(\mu, \lambda) \in C_{c}$ we have $a\left(F_{(\mu, \lambda)}\right)=c$ and for $(\mu, \lambda) \in C_{d}$ we have $b\left(F_{(\mu, \lambda)}\right)=d$.

For instance for $a_{0}=\underline{01}, b_{0}=\underline{10}$, we have:

$$
\begin{aligned}
& \underline{a_{1}}=\underline{0011}, a_{2}=\underline{00101101}, a_{3}=\underline{0010110011010011}, \\
& a_{4}=\underline{00101100110100101101001100101101}, \quad \text { abd } \\
& \underline{b_{1}}=\underline{1100}, b_{2}=\underline{11010010}, b_{3}=\underline{1101001100101100}, \\
& b_{4}=\underline{1101001100101101010110011010010}, C_{00101101 .}
\end{aligned}
$$


Associated to this doubling period we have the following picture.

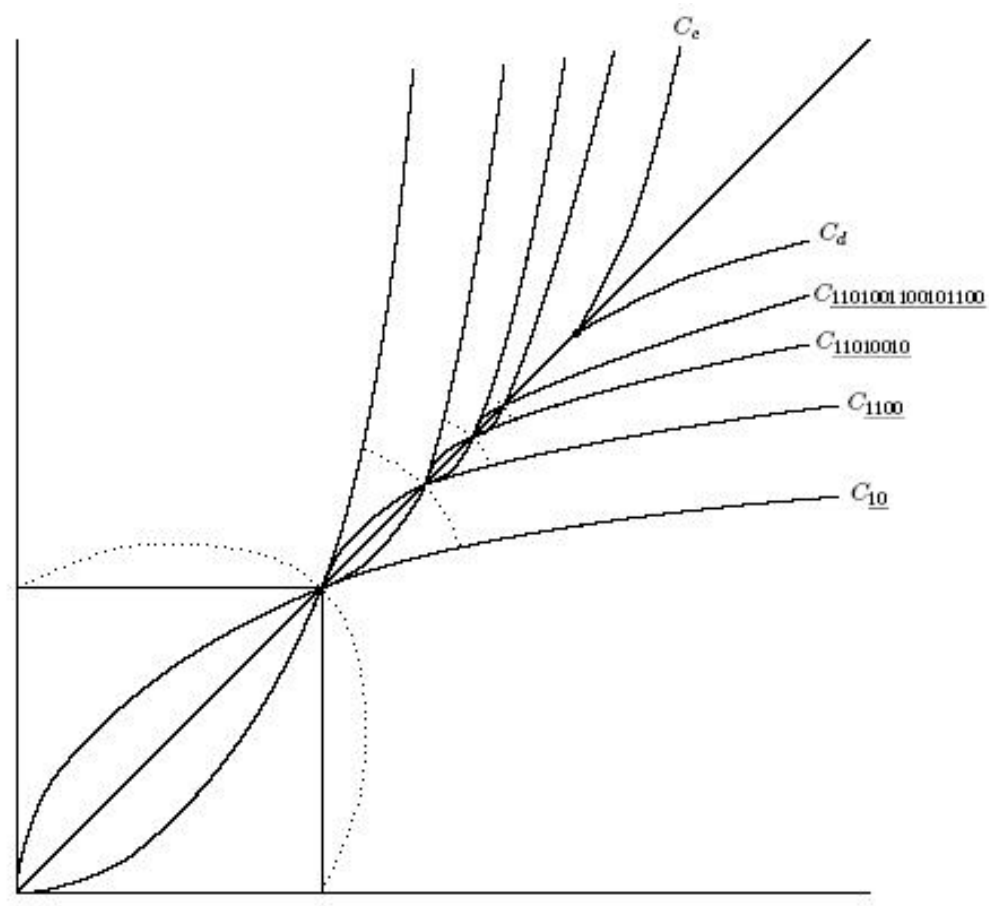

\subsection{Tails}

Lemma 14. a) Assume $a \in A_{\infty}^{\infty}$ and $d \in M_{a x}$ is a non-periodic sequence such that $C_{a} \cap C_{d} \neq \emptyset$ (see figre 25).

There is a curve $C_{a_{-} d}$, which "born" in $\{P(a, d)\}=C_{a} \cap C_{d}$, tangent to $C_{d}$ such that $P(a, d) \in \overline{C_{a_{-}}}$and $(\mu, \lambda) \in C_{a_{-} d}$ imply $a\left(F_{(\mu, \lambda)}\right)=$ $a_{-} d$.

b) Assume $b \in \beta_{\infty}^{\infty}$ and $c \in M a x_{2}$ is a non-periodic sequence such that 
$C_{c} \cap C_{b} \neq \emptyset$.

There is a curve $C_{b_{+} c}$, which "born" in $\{P(c, d)\}=C_{c} \cap C_{b}$, tangent to $C_{c}$ at this point such that $P(c, d) \in \overline{C_{b_{+}}}$and $(\mu, \lambda) \in C_{b_{+} c}$ imply $b\left(F_{(\mu, d)}\right)=b_{+} c$ (see figure 26).

We let the proof of this lemma to the reader.

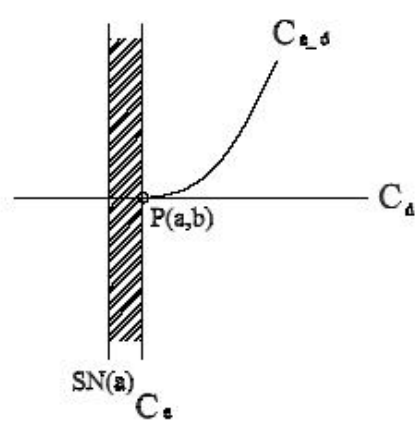

Figure 25:

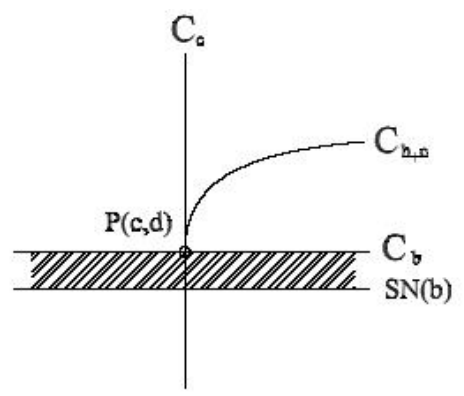

Figure 26:

The figures (26)and (27) displays these facts.

\subsection{Entropy zero}

In this section we will make (following [23]) some remarks concerning the set

$$
E Z=\left\{(\mu, \lambda) ; h\left(F_{(\mu, \lambda)}\right)=0\right\} .
$$

Here, $h(f)$ means the topological entropy of the map $f \in D M_{0}$.

a) Assume $a \in A_{\infty}^{\infty}, b=b(a)$. We have

$$
\operatorname{int}\left(L_{a} \cap R_{b}\right) \subset \operatorname{int}(E Z)
$$


In fact, in this situation the set $\Sigma_{a, b}$ is formed by the iterations $\sigma^{i}(a), i=$ $1, \cdots, \# \operatorname{per}(a)-1$ and their preimages under the map $\sigma$.

b) Assume $a \in A_{\infty}^{\infty}, b=b(a)$ and $\{P(a, b)\}=C_{a} \cap C_{b}$ then $P(a, b) \in$ $\operatorname{int}(E Z)$.

In fact, there is a neighborhood of the point $P(a, b)$, which is covered by the union $L_{a} \cup L_{a_{-} b} \cup\left\{L_{c} ; c \in A(a, b) \cup B(a, b)\right\} \cup\left\{C_{c} ; c \in \overline{A(a, b) \cup B(a, b)}\right\}$. ( here $A(a, b)$ and $B(a, b)$ where defined in 4.3.1. It is clear that for any $c \in \overline{A(a, b) \cup B(a, b)}\}$ we have that the set $\Sigma_{c, d}$ is formed by the iterations $\sigma^{i}(c) i=1, \cdots, \# \operatorname{per}(c)-1$ and their preimages under the map $\sigma$, here $d=\sup \left\{\sigma^{i}(c) ; i \in N\right\}$.

c) Assume $a \in A_{\infty}^{\infty}, b=b(a)$ then

$$
\left.\operatorname{int}\left(L_{a_{-\underline{b}}}\right) \cup R_{b_{+} \underline{a}}\right) \subset \operatorname{int}(E Z) \text {. }
$$

In fact, if $(\mu, \nu) \in \operatorname{int}\left(L_{a_{-} \underline{b}}\right)$ we have that $a(\mu, \nu)=a$ and $b(\mu, \nu)=$ $\sup \left\{\sigma^{i}(a) i \in N\right\}$.

d) Assume $a \in A_{\infty}^{\infty}, b=b(a)$ then

$$
\overline{L_{a}} \cap C_{b_{+} \underline{a}} \subset E Z \backslash \operatorname{int}(E Z)
$$

and

$$
\overline{R_{b}} \cap C_{a_{-} \underline{b}} \subset E Z \backslash \operatorname{int}(E Z)
$$

In fact, given any $(\mu, \nu) \in \overline{L_{a}} \cap C_{b_{+} a}$ we have that there is a small neighborhood $V(\mu, \nu)$ of the point $(\mu, \nu)$ which is divided by the curve $C_{b_{+} \underline{a}}$ in two disjoint pieces, one of them, included in $\operatorname{int}(E Z)$ and the other included in the interior of the positive entropy set.

All the next results, in this section, follows in a similar way.

e) Let $a \in A_{\infty}^{\infty}, b=b(a)$ and $\left(P(a, b), Q_{a_{-}}\right) \subset C_{b}$ be the open interval whose boundary points are $P(a, b)$ and $Q_{a_{-} b}$ then

$$
\left(P(a, b), Q_{a_{-} b}\right) \subset \operatorname{int}(E Z) .
$$

f) Let $a \in A_{\infty}^{\infty}, b=b(a)$ and $\left(P(a, b), Q_{b_{+}}\right) \subset C_{a}$ be the open interval whose boundary points are $P(a, b)$ and $Q_{b_{+} \underline{a}}$ then $\left(P(a, b), Q_{b_{+} \underline{a}}\right) \subset$ $\operatorname{int}(E Z)$.

g) Also, we have

$$
\{(\mu, 0) ; 0<\mu<1\} \subset \operatorname{int}(E Z)
$$

and

$$
\{(0, \nu) ; 0<\nu<1\} \subset \operatorname{int}(E Z) .
$$

Moreover:

$$
(1,0) \in[E Z \backslash \operatorname{int}(E Z)]
$$

and

$$
(0,1) \in[E Z \backslash \operatorname{int}(E Z)] \text {. }
$$

h) Let $a \in A_{\infty}^{\infty}, b=b(a)$. For any $(\mu, \nu)$ such that

$$
a\left(F_{(\mu, \lambda)}\right) \leq a_{-} \underline{b} \quad \text { and } \quad b\left(F_{(\mu, \lambda)}\right)>b
$$


or

$$
a\left(F_{(\mu, \lambda)}\right)<a_{-} \underline{b} \quad \text { and } \quad b\left(F_{(\mu, \lambda)}\right) \geq b
$$

we have that $(\mu, \lambda) \in \operatorname{int}\left\{(\mu, \lambda) ; h\left(F_{(\mu, \lambda)}\right)>0\right\}$

i) Let $a \in A_{\infty}^{\infty}, b=b(a)$. For any $(\mu, \nu)$ such that

$$
a\left(F_{(\mu, \lambda)}\right) \leq a \quad \text { and } \quad b\left(F_{(\mu, \lambda)}\right)>b_{+} \underline{a}
$$

or

$$
a\left(F_{(\mu, \lambda)}\right)<a \quad \text { and } \quad b\left(F_{(\mu, \lambda)}\right) \geq b_{+} \underline{a}
$$

We have that $(\mu, \lambda) \in \operatorname{int}\left\{(\mu, \lambda) ; h\left(F_{(\mu, \lambda)}\right) \neq 0\right\}$.

j) Let $a \in A_{\infty}^{\infty}, b=b(a)$. Denote by $B(a, b) \in R^{2}$ the point where the curves $C_{a}$ and $C_{b}$ born. Let $S N(a, b)=C_{a} \cap S N(b) ; \overline{S N}(a, b)=C_{b} \cap S N(a)$. We have that the intervals $\left[B(a, b), S N(a, b)\left[\subset C_{a}\right.\right.$ and $[B(a, b), \overline{S N}(a, b)[$ are contained in $\operatorname{int}\left(\left\{(\mu, \lambda) ; h\left(F_{(\mu, \lambda)}\right)=0\right\}\right)$.

k) Let

$$
C_{\underline{0}}=\left\{\left(\frac{1+\sqrt{1+4 \nu}}{2}, \nu\right) ; \quad \nu \geq 0\right\}
$$

and

$$
C_{1}=\left\{\left(\mu, \frac{1+\sqrt{1+4 \mu}}{2}\right) ; \quad \mu \geq 0\right\}
$$

We have,

$$
\left(C_{\underline{0}} \backslash\{(1,0)\}\right) \cup\left(C_{\underline{1}} \backslash\{(0,1)\}\right) \subset \operatorname{int}\left(\left\{(\mu, z) ; h\left(F_{(\mu, \lambda)}\right) \neq 0\right\}\right) .
$$

l) Let

$$
\begin{gathered}
L_{\underline{0}}=\left\{(\mu, \nu) ; \mu \geq \frac{1+\sqrt{1+4 \nu}}{2}, \nu \geq 0\right\}, \\
R_{\underline{1}}=\left\{(\mu, \nu) ; \nu \geq \frac{1+\sqrt{1+4 \nu}}{2}, \mu \geq 0\right\}, \\
L_{0 \underline{1}}=\{(\mu, \nu), a(\mu, \nu)=0 \underline{1}\},
\end{gathered}
$$

and

$$
R_{1 \underline{0}}=\{(\mu, \nu), b(\mu, \nu)=1 \underline{0}\} .
$$

We have

$$
\operatorname{int}\left(L_{0} \backslash R_{1 \underline{0}}\right) \cup \operatorname{int}\left(R_{\underline{1}} \backslash L_{0 \underline{1}}\right) \subset \operatorname{int}\left(\left\{(\mu, \nu) ; h\left(F_{(\mu, \lambda)}\right) \neq 0\right\}\right) .
$$

From all of the above results it is clear that $E Z$ is an arc-connected set. We do not know if it is locally connected. 


\subsection{Non-zero measure for the Bifurcation}

The result in this subsection is a consequence of a result proved by Rovella in [36]. We established it here only for the sake of completness.

Let $a \in M_{i n}$ and $b \in M a x_{2}$ be eventually periodic sequences such that $C_{a} \cap C_{b} \neq \emptyset$. Let $C_{a} \cap C_{b}=\{P(a, b)\}$ and $U(a, b)$ be any neighborhood of $P(a, b)$. We have

Lemma 15. $m\left(\left\{(\mu, \nu) \in U(a, b) ; F_{(\mu, \nu)}\right.\right.$ is not $\left.\left.A x A\right\}\right)>0$.

As a consequence of this result we get

Corollary 2. Let $(\mu, \nu) \in(E Z \backslash \operatorname{int}(E Z))$ and $U(\mu, \lambda)$ be any neighborhood of $(\mu, \lambda)$. We have

$$
m\left(\left\{(\alpha, \beta) \in U(\mu, \lambda) ; F_{(\alpha, \beta)} \text { is not } A x . A\right\}\right)>0 .
$$

Proof: $(\mu, \lambda)$ can be approximated by a sequences $\left(\mu_{n}, \nu_{n}\right)$ as in the Lemma 15.

\subsection{Tales in $L_{\underline{0}}$ and $R_{\underline{1}}$}

a) Associated to $b=1 \underline{0}$ there is a curve $C_{10} \subset L_{0}$ such that $(1,0) \in$ $\overline{C_{10}}$; this curve is tangent to $C_{\underline{0}}$ at $(1,0)$ and $(\mu, \nu) \in \bar{C}_{1 \underline{0}}$ imply $b\left(F_{(\mu, \lambda)}\right)=$ $1 \underline{0}$.

b) Associated to $a=0 \underline{1}$ there is a curve $C_{0 \underline{1}} \subset R_{\underline{1}}$ that satisfy $(0,1) \in$ $\overline{C_{0 \underline{1}}}$; it is tangent to $C_{\underline{1}}$ and $(0,1) ;(\mu, \nu) \in C_{0 \underline{1}}$ imply $a\left(F_{(\mu, \lambda)}\right)=0 \underline{1}$;

c) Let $\left.\varphi_{1 \underline{0}}:\right] 1, \infty\left[\rightarrow R\right.$ denote the map that satisfy $\operatorname{Graph}\left(\varphi_{1 \underline{0}}\right)=C_{1 \underline{0}}$. We have

$$
\left.\left\{(\mu, \nu) ; b\left(F_{(\mu, \lambda)}\right)\right\}=1 \underline{0}\right\}=\left\{(\mu, \nu) ; 0<\nu \leq \varphi_{1 \underline{0}}(\mu) ; \mu>1\right\} .
$$

d) Let $\left.\varphi_{0 \underline{1}}:\right] 1, \infty\left[\rightarrow R\right.$ denote the map that satisfy $\operatorname{Graph}\left(\varphi_{01}\right)=C_{0 \underline{1}}$. We have

$$
\left\{(\mu, \nu) ; a\left(F_{(\mu, \lambda)}\right)=0 \underline{1}\right\}=\left\{(\mu, \nu) ; 0<\mu \leq \varphi_{0 \underline{1}}(\nu), \quad \nu>1\right\} .
$$

e) Let $b \in B_{\infty}^{\infty}$ and $\{P(\underline{0}, b)\}=C_{\underline{0}} \cap C_{b}$.

There is a curve $C_{b_{+} \underline{0}} \subset L_{0}$ such that $P(\underline{0}, b) \in \overline{C_{b_{+}}} ; C_{b_{+} \underline{0}}$ is tangent to $C_{\underline{0}}$ at $P(\underline{0}, b)$ and $(\mu, \bar{\lambda}) \in \overline{C_{b_{+}} \underline{0}}$ imply $b\left(F_{(\mu, \lambda)}\right)=b_{+} \underline{0}$.

f) Let $P(\underline{0}, b)=\left(\mu_{0, b}, \nu_{u 0, b}\right)$ and $\left.\varphi_{b_{+} \underline{0}}:\right] \mu_{\underline{0}, b}, \infty[\rightarrow R$ be the map that satisfy $\operatorname{Graph}\left(\varphi_{b_{+}}\right)=\bar{C}_{b_{+} \underline{0}}$.

Let $\varphi_{b}:\left[\mu_{0}, \infty\right] \rightarrow R$ be the map that satisfy $\operatorname{Graph}\left(\varphi_{b}\right)=L_{\underline{0}} \cap C_{b}$.

Clearly $\varphi_{b}(\mu)<\varphi_{b_{+} \underline{0}}(\mu), \mu>\mu_{\underline{0}, b}$. 
We have $\left\{(\mu, \nu) ; b\left(F_{(\mu, \lambda)}\right)=b_{+} \underline{0}\right\}=\left\{(\mu, \nu) ; \varphi_{b}(\mu)<\nu \leq \varphi_{b_{+} \underline{0}}(\mu)\right\}$.

g) Let $a \in A_{\infty}^{\infty}$ and $\{P(a, \underline{1})\}=C_{a} \cap C_{\underline{1}}$. There is a curve $C_{a_{-}} \subset R_{\underline{1}}$ such that $P(a, \underline{1}) \in \overline{C_{a_{-}}} ; C_{a_{-}}$is tangent to $C_{\underline{1}}$ at $P(a, \underline{1})$ and $(\bar{\mu}, \lambda) \bar{\epsilon}$ $C_{a_{-}}$imply $a\left(F_{(\mu, \lambda)}\right)=\bar{a}_{-}$.

h) Let $P(a, \underline{1})=\left(\mu_{a, \underline{1}}, \nu_{a, \underline{1}}\right)$ and $\left.\varphi_{a_{-}}:\right] \nu_{a, \underline{1}}, \infty\left[\rightarrow R, \varphi_{a}:\left[\nu_{a, \underline{1}}, \infty[\rightarrow R\right.\right.$ be the maps that satisfies:

$$
\operatorname{Graph}\left(\varphi_{a_{-}}\right)=C_{a_{-} \underline{1}} \quad \text { and } \operatorname{Graph}\left(\varphi_{a}\right)=R_{\underline{1}} \cap C_{a} .
$$

Clearly $\varphi_{a}(\nu)<\varphi_{a_{-}}(\nu)$,for any $\nu>\nu_{a_{-}, \underline{1}}$. $\left.\nu_{a, 1}\right\}$.

We have $\left\{(\mu, \nu) ; a\left(F_{(\mu, \nu)}\right)=a_{-} \underline{1}\right\}=\left\{(\mu, \nu) ; \varphi_{a}(\nu)<\mu \leq \varphi_{a_{-}}(\nu), \quad \nu>\right.$

\subsection{Bifurcations in $R_{\underline{1}} \cup L_{\underline{0}}$}

In the same way as was proved in [19] or as in [34] we can prove.

Lemma 16. $m\left(\left\{(\mu, \nu) \in R_{\underline{1}} \cup L_{\underline{0}} ; F_{(\mu, \nu)}\right.\right.$ do not satisfy $\left.\left.A x . A\right\}\right)=0$.

\subsection{Density of Axiom A Systems.}

A consequence of our's and Rovella's result is:

Lemma 17. $\left\{(\mu, \nu) ; F_{(\mu, \nu)}\right.$ satisfy $\left.A x . A\right\}$ is a dense set in $R_{0}^{+} \times R_{0}^{+}$.

\subsection{The Global Picture}

Taking together all the results in this section the global picture of the bifurcation diagramme is as in figure 27 .

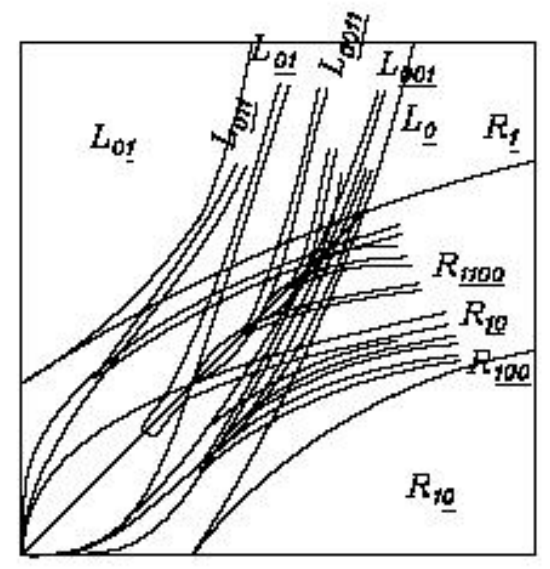

Figure 27: 


\section{The general case}

Let us consider the five parameter family of maps $F_{\left(\alpha, c_{1}, c_{2}, \mu, \nu\right)}:(R \backslash\{0\}) \rightarrow$ $R, \mu \geq 0, \nu \geq 0 \alpha>1, c_{1}>0, c_{2}>0$ in $D M_{0}$ given by:

$$
F_{\left(\left(\alpha, c_{1}, c_{2}, \mu, \nu\right)\right.}(x)=\left\{\begin{array}{l}
-\mu+c_{1} x^{\alpha}, \quad x>0 \\
\nu-c_{2}(-x)^{\alpha}, \quad x<0
\end{array}\right.
$$

We have to observe that all the previous results, for the quadratic family, are (still) true when we fix generic values of the parameter $\alpha, c_{1}$ and $c_{2}$. That is, the bifurcation diagramme associated to any generic two parameter family $F_{(\mu, \nu)}=F_{\left(\alpha, c_{1}, c_{2}, \mu, \nu\right)}$ is, up to an homeomorphism, the same as the given for the quadratic family.

\section{Application to Geometric Vector fields}

Assume $X_{0}$ : $U \subset R^{3} \rightarrow R^{3}$ is a three dimensional vector field that satisfies:

a) $0 \in U$ is an hyperbolic singularity of the vector field $X$, whose eigenvalues satisfies $-\lambda_{1}<-\lambda_{2}<0<\lambda_{3}$;

b) The components, $\gamma_{1}$ and $\gamma_{2}$, of the set $\left(W_{0}^{u} \backslash\{0\}\right)$ satisfies $\gamma_{1} \subset W_{0}^{s}$, $\gamma_{2} \subset W_{0}^{s}$;

c) There is a transversal section, $\Sigma \subset U$, such that: $\Sigma$ is transversal to $W_{0}^{s}$ and $\Sigma \backslash W_{0}^{s}=\Sigma_{1} \cup \Sigma_{2}$ are two disjoint sets in $\Sigma$ that satisfies:

$$
\pi_{1}\left(\Sigma_{1}\right) \subset \Sigma_{1} \quad \text { and } \quad \pi_{2}\left(\Sigma_{2}\right) \subset \Sigma_{2}
$$

where $\pi_{i}: \Sigma_{i} \rightarrow \Sigma, i=1,2$ is the first return map associated to the respective cross section.

Let $\mathcal{U}_{0} \subset X^{r}\left(U, R^{3}\right)$ be a neighborhood of the vector field $X_{0}$ in $X^{r}\left(U, R^{3}\right)=$ $\left\{X: U \subset R^{3} \rightarrow R^{3} ; \quad X\right.$ is $\left.C^{r}\right\}$ with the usual $C^{r}$-topology.

In the above conditions there are codimension one submanifold, $\mathcal{N}_{1}, \mathcal{N}_{2} \subset$ $\mathcal{U}_{0}$; such that:

a) $Y \in \mathcal{N}_{i}$ if and only if $\gamma_{i}(Y) \subset W_{\sigma(Y)}^{s}$ here $\sigma(Y)$ is the hyperbolic singularity, near 0 , that correspond to the vector field $Y$. Also, $\left(W_{\sigma(Y)} \backslash\{\sigma(Y)\}\right)=\gamma_{1}(Y) \cup \gamma_{2}(Y)$, where $\gamma_{i}(Y)$ is the natural extension of the component $\gamma_{i}$;

b) $\left(\mathcal{U}_{0} \backslash \mathcal{N}_{i}\right)=\mathcal{U}_{1}^{i} \cup \mathcal{U}_{2}^{i}$ where $Y \in \mathcal{U}_{1}^{1}$ if and only if $\pi_{1}\left(\Sigma_{1}\right) \subset \Sigma_{1}$ and $Y \in \mathcal{U}_{2}^{2}$ if and only if $\pi_{2}\left(\Sigma_{2}\right) \subset \Sigma_{2}$ and

c) $\mathcal{N}_{1}$ is transversal to $\mathcal{N}_{2}$. 
Finally we will assume that $\Sigma$ can be foliated by one-dimensional submanifolds $\left\{\mathcal{F}_{x} ; \quad x \in \Sigma\right\}$ such that

(i) $x \in W_{Y}^{s} \cap \Sigma$ imply $\mathcal{F}_{x}=W_{Y}^{s} \cap \Sigma$ and

(ii) $\pi_{i}\left(\mathcal{F}_{x}\right) \subset \mathcal{F}_{\pi_{i}(x)}, x \in \Sigma_{i}$.

In this situation the interesting part of the bifurcation theory, for elements in $\mathcal{U}_{0}$, is located in $\mathcal{U}_{1}^{2} \cap \mathcal{U}_{2}^{1}$. In this set we can apply the results in Sections 3,4 and 5 to obtain, bis a bis, similar results for generic two parameter families of vector fields in $\mathcal{U}_{0}$.

\section{ACKNOWLEDGMENTS}

Part of this paper is an outgrowth of research during a visit of the authors to ICTP (Trieste - Italy), a visit of Rafael Labarca to IMPA-Brazil (as part of an MATHAMSud and PRONEX project)and a visit of Carlos Moreira to the Universidad de Santiago de Chile. We thanks to these institutions for their support and hospitality while preparing this paper. We also would like to thanks to Marcelo Viana and to an unknown referee for helpful comment concerning a previous version of this paper. We also would like to thanks to the MATHAMSUD project 09MATH02 by its support.

\section{References}

[1] Afraimovich V. S., Bykov V.V., Shil'nikov L.P The origin and structure of the Lorenz attractor Dokl. Akad. Nauk SSSR 234, (1977), No 2, pp. 336-339, (1977).

[2] Afraimovich V. S., Bykov V.V., Shil'nikov L.P. On structurally stable attracting limit sets of Lorenz attractor type Trans. Mosc.Math. Soc. 44, pp. 153-216 (1983). pp. 431-443, (1990).

[3] Arnold V. I. Small Denominators. I. On the mapping of the circumference onto itself. Amer. Math. Soc. Transl. $2^{\text {end }}$ Ser. Vol. 46, pp. 213-284, (1965).

[4] Balibrea F., Jiménez López V. A characterization of chaotic functions with entropy zero via their maximal scrambled sets. Math. Bohem. 120, $N^{o}$ 2, pp. 293-298, (1998).

[5] Bamón,R.; Labarca, R.; Mañé R.; Pacifico, M. J. The explosion of singular cycles Publ. Math. IHES vol 78, pp. 207-232, (1993). 
[6] Boyland P. Bifurcations of Circle Maps: Arnold's Tongues, Bistability and Rotation Intervals Comm. Math. Phys. 106, pp. 353-381, (1986).

[7] Brucks K. M., Misiurewicz M., Tresser Ch. Monotonicity properties of the family of trapezoidals maps Comm. Mat. Phys. 137, pp. 1-12(1991).

[8] Campbell D. K., Galeeva R., Tresser CH., Uherka D.J. Piecewise linear models for the quasiperiodic transition to chaos Chaos 6(2), pp. 121-154, (1996).

[9] De Melo W. , Martens M. Universal Models for Lorenz maps Ergod. Th. \& Dynam. Sys., 21, pp. 833-860, (2001).

[10] deMelo W., Van Strien S. Lectures on One Dimensional Dynamics Springer Verlag. (1993).

[11] Galeeva R., Martens M., Tresser Ch. Inducing, slopes and conjugacy classes Israel Journal of Math. 99, pp. 123-147 (1997).

[12] Galeeva R., Van Strien S. Which families of l-modals maps are full TAMS, vol 348, \#8, pp. 3215-3221, (August 1996 ).

[13] Gambaudo, J-M., Tresser C. Dynamique régulière ou chaotique. Applications du cercle ou l'intervalle ayant une discontinuité. C.R. Acad. Sc. Paris, t. 300, Serie I, $\mathrm{n}^{0}$ 10, pp. 311-313 (1985).

[14] Graczyk J., Swiatek G. Generic Hiperbolicity in the logistic family Ann. of Math. 146, pp. 1-52 (1997).

[15] Guckenheimer J. A Strange, Strange AttractorIn :Hopf Bifurcations and its applications J. E. Marsden and M. McCracken Eds. pp. 368381. Springer Verlag. Berlin. 1976.

[16] Guckenheimer J., Williams R. F. Structural Stability of Lorenz Attractors Publ. Math. IHES 50, pp. 59-72, (1979).

[17] Herman M. Mesure de Lebesgue et nombre de rotation. Lect. Not. in Math. 597, pp 271-293 ( 1977).

[18] Hubbard J. H., Sparrow C.T. The classification of topologically expansive Lorenz maps Comm. Pure and App. Math. XLIII, pp 431-443, (1990).

[19] Labarca, R. Bifurcation of Contracting Singular Cycles Ann. Scient. Ec. Norm. Sup. 4serie,t. 28, pp. 705-745, (1995).

[20] Labarca, R.A note on the topological classification of Lorenz maps on the interval London Mathematical Society. Lecture Notes Series 279, pp. 229-245.

[21] Labarca,R.; Moreira C. Bifurcation of the Essential Dynamics of Lorenz Maps of the real Line and the Bifurcation Scenario for the Linear family Scientia Ser A Math. Sci (N-S) vol. 7, pp. 13-29, (2001). 
[22] Labarca, R.; Moreira C. Bifurcation of the Essential Dynamics of Lorenz Maps and applications to Lorenz Like Flows: Contributions to the study of the Expanding Case. Bol. Soc. Bros. Mat. (N-S) Vol. 32 ,pp. 107-144, (2001).

[23] Labarca, R.; Moreira C. Essential Dynamics for Lorenz Maps on the real line and Lexicographical World. Ann. de L'Institut H. Poincar Anal. non Linneaire vol. 23, pp. 683-694 (2006).

[24] Labarca R., Plaza S. Bifurcations of discontinuous maps of the interval and palindromic numbers. Boletín de la Sociedad Matemática Mexicana(3) Vol 5., pp. 87-104. (2001).

[25] Labarca R., Vásquez L. On the Characterization of the Kneading Sequences Associated to Injective Lorenz Maps of the Interval and to Orientation Preserving Homeomorphisms of the Circle. Accepted for publication in the Boletín de la Sociedad Matemática Mexicana(3). (2010).

[26] Labarca R., Vásquez L. On the Characterization of the Kneading Sequences Associated to Lorenz Maps of the Interval. Preprint (2010).

[27] Lorenz E. N. Deterministic non-periodic flow J. Atmos. Sci. 20, pp. 130-141, (1963).

[28] MacKay R. S., Tresser Ch. Boundary of topological chaos for bimodal maps of the interval J. London Math. Soc. (2) 37, pp. 164-181, (1988).

[29] MacKay R., Tresser Ch. Some flesh on the skeleton: the bifurcation structure of bimodals maps Physica D 27, pp. 412-422, (1987).

[30] Mañé R. Ergodic Theory and Differentiable Dynamics. Springer Verlag. (1987).

[31] Martens M., Tresser Ch. Forcing of periodic orbits for interval maps and renormalization of piecewise affine maps Proc. Am. Math. Soc. Vol. 124, Number 9, pp. 2863-2870, (1996).

[32] Sands D., Nowicki T. Non-uniform hiperbolicity and universal bounds for S-unimodal maps Inven. Math. 132, pp. 633-680, (1998).

[33] Otero-Espinar M. V., Tresser Ch. Global Complexity and Essential Simplicity: A conjectural picture of the boundary of chaos for smooth endomorphisms of the interval Physica D 39, pp. 163-168, (1989).

[34] Pacifico, M. J., Rovella A. Unfolding Contracting Singular Cycles Ann. Scient. Ec. Norm. Sup. 4serie t.26, pp. 691-700, (1993).

[35] Ringland J., Tresser Ch. A genealogy for finite kneading sequences of bimodal maps on the intervalTAMS, Vol. 347, \#12, pp 4599-4624, (December 1995).

[36] Rovella A. The dynamics of the perturbations of the contracting Lorenz Attractor Bull. Soc. Bras. Mat. Vol 2, pp. 233-259 )(1993). 
[37] Saint Pierre M. Topological and measurable dynamics of Lorenz maps Dissertationes Mathematicae 1999, POLSKA AK. NAUK, INST. MAT.

[38] Swiateck G. Rational Rotation Numbers for mapps of the circle Comm. Math. Phys. 119, pp. 109-128, (1988), .

[39] Tresser Ch. Nouveaux types de transitions vers une entropie topologique positive CRAS, t 296, Serie I, pp. 729-732, (1983).

[40] Williams R. F. The structure of Lorenz Attractors. In Turbulence Seminar Berkeley 1976/1977 P. Bernard, T. Ratiu (Eds.), Springer-Verlag. New York, Heidelberg, Berlin, pp. 94-112.

R. Labarca

Departamento de Matematica y Ciencias de la Computación

Universidad de Santiago de Chile

Casilla 307 Correo 2

Santiago

e-mail : rafael.labarca@usach.cl

and

C. Moreira

Éstrada Dona Castorina 110

CEP. 22460-320

Jardim Botanico

Rio de Janeiro

Brazil :

e-mail : gugu@impa.br 